\title{
Lung Cancer Risk in Painters: A Meta-Analysis
}

\author{
Risco de Câncer de pulmão em pintores: Uma meta-análise
}

\author{
Neela Guha ${ }^{1}$ \\ Franco M erletti ${ }^{2}$ \\ Nelson Kyle Steenland ${ }^{3}$ \\ Andrea Altieri 4 \\ Vincent Cogliano ${ }^{1}$ \\ Kurt Straif ${ }^{1}$
}

*Supplemental M aterial is available online (doi: 10.1289/ ehp.0901402.S1 viahttp://dx.doi.org/) 'Section of IARC M onographs, International Agency for Research on Cancer. 150 cours Albert Thomas 69372 Lyon cedex 8 France. guhan@iarc.fr ${ }^{2}$ Unit of Cancer

Epidemiology, Department of Biomedical Sciences and Human Oncology,

University of Turin, Turin,

Italy.

${ }^{3}$ Department of

Environmental and

Occupational $\mathrm{H}$ ealth,

Rollins School of Public

Health, Emory University,

Atlanta, Georgia, USA. ${ }^{4}$

Emerging Risks U nit,

European Food Safety

Authority, Parma, Italy.
Abstract Weconducted a meta-analysis to quantitatively compare the association between occupation as a painter and the incidence or mortality from lung cancer. PubM ed and the reference lists of pertinent publications were searched and reviewed. For the meta-analysis, we used data from 47 independent cohort, record linkage, and case-control studies (from a total of 74 reports), including $>11,000$ incident cases or deaths from lung cancer among painters. Three authors independently abstracted data and assessed study quality. The summary relative risk (meta- $R R$, random effects) for lung cancer in paint-ers was 1.35 [ $95 \%$ confidenceinterval ( $\mathrm{Cl}$ ), 1.29-1.41; 47 studies] and 1.35 (95\% Cl, 1.21-1.51; 27 studies) after controlling for smoking. The relative risk was higher in never-smokers (meta-RR $=2.00 ; 95 \%$ $\mathrm{Cl}, 1.09-3.67$; 3studies) and persisted when restricted to studies that adjusted for other occupational exposures (meta- $\mathrm{RR}=1.57 ; 95 \% \mathrm{Cl}, 1.21$ 2.04; 5 studies). These results support the conclusion that occupational exposures in painters are causally associated with the risk of lung cancer. Key words Epidemiology, Lung cancer, M etaanalysis, Painter
Resumo Conduziu-se uma meta-análise para comparar quatitativamente a associação entre 0 trabalho de pintor e a incidência ou mortalidade por câncer de pulmão. PubM ed elistas de referência de publicações pertinentes foram pesquisadas erevisadas. Para a análise, foram usados dados de 47 estudos de caso, coorte independente e ligação de dados (deum total de 74 relatórios), incluindo $>11$ mil casos de incidentes ou morte por câncer de pulmão entre pintores. Três autores coletaram dados e avaliaram a qualidade de estudo. 0 risco relativo (meta- $R R$, efeitos aleatórios) de câncer de pulmão em pintores foi de 1,35 [ 95\% intervalo de confiança (IC), 1,29-1,41; 47 estudos] e 1,35 ( $95 \%$ IC, 1,21-1,51; 27 estudos) depois de se destacar os fumantes. 0 risco relativo foi maior naqueles quenunca fumaram (meta- $R R=2.00 ; 95 \%$ $\mathrm{Cl}, 1,09-3,67 ; 3$ estudos) e persistiu quanto restringido a estudos que foram ajustados para outras exposições ocupacionais (meta- $R R=1,57$; $95 \% \mathrm{Cl}, 1,21-2,04 ; 5$ estudos). Estes resultados sustentam a conclusão de que exposi ções ocupacionais em pintores são causadamenteassociada com o risco de câncer de pulmão.

Palavras-chave Epidemiologia, Câncer de pulmão, M eta-análise, Pintor 
Lung cancer is the most common cancer diagnosis worldwide and is the major cause of cancer mortality, particularly among men. The International Agency for Research on Cancer (IARC) estimated that there were $>900,000$ new cases of lung cancer each year among men and $>330,000$ among women (IARC 2001, 2003). A pproximately $90 \%$ of thelung cancer burden in developed countries is attributed to smoking, which acts either independently or synergistically with other occupational, lifestyle, or hereditary risk fac-tors (Boffetta and Trichopoulos 2002; Peto et al. 1994). Several agents encountered in the occupational setting, such as asbestos, polycyclic aromatic hydrocarbons, arsenic, beryllium, cadmium, chromium (VI), and nickel compounds, areestablished carcinogens that target the lung (IARC 2008).

An increased incidence and mortality from lung cancer has been observed in painters, an occupation that employs several million people worldwide (IARC 1989). This has led IARC to classify occupational exposureasa painter as"carcinogenic to humans" (Group1) (IARC 1989, in press; Straif et al. 2007). Painters are exposed to many known and suspected lung carcinogens through inhalation or dermal con-tact (IARC 1989; Siemiatycki et al. 2004), such as talc containing asbestos fibers, chromium VI compounds, chlorinated solvents, and cadmium compounds (IARC 1987, 1995, 1999, in press; Straif et al. 2009), although the specific caus-ative agents have not yet been identified.

Cohort and record linkagestudies demonstrating a relatively consistent increased incidence and mortality from lung cancer among painters [Alexander et al. 1996; Boiceet al. 1999; D ubrow and Wegman 1984; Dunn and Weir 1965; Enterlineand M cKiever 1963; Gubéran et al. 1989; Guralnick 1963; H rubec et al. 1995; Logan 1982; M enck and Henderson 1976; Office of Population Censuses and Surveys(OPCS) 1958, 1971, 1978, 1986, 1995; Petersen and Milham 1980; Pukkala 2009; van Loon et al. 1997; Whorton et al. 1983] have supported theIARC Group 1 classification, although potential confounding by tobacco smoking could not be ruled out in several of these studies. (Here we refer to record linkage studies as a subset of cohort studies where two databases are linked, such as a cohort of painters derived from census data and national mortality data, with only minimum demographic information availablefor the cohort.) Case-control studies have also shown that occupational exposure as a painter is a risk factor for lung cancer (Bethwaiteet al. 1990; Bouchardy et al. 2002; Breslow et al. 1954; DeStefani et al. 1996; Finkelstein 1995; M ilneet al. 1983; Pohlabeln et al. 2000; Wynder and Graham 1951), al beit some-what less consistently (Baccarelli et al. 2005; M orabia et al. 1992; M uscat et al. 1998; Vineis et al. 1988; WünschFilho et al. 1998), and the increased risk persisted after adjusting for the potential confounding by smoking (Brüske-H ohlfeld et al. 2000; Coggon et al. 1986; Decoufléet al. 1977; H outen et al. 1977; Jahn et al. 1999; Kjuus et al. 1986; Lerchen et al. 1987; Richiardi et al. 2004; Ronco et al. 1988; Viadana et al. 1976; Williams et al. 1977).

To assess the risk of lung cancer associated with occupational exposure as a painter, we conducted a meta-analysis of cohort, record linkage, and case-control studies to quantitatively compare the results of the different study designs and the potential confounding effect of smoking (by restricting to never-smokers), as well as other analyses to support thecausal association. A thorough discussion of the individual studies included in the meta-analysis is not presented here but was summarized in the IARC M onographs (IARC 1989, in press). All of thestudies reviewed, including the new studies published since the IARC $M$ onographs, are summarized in Supplemental M aterial, Tables1-3, availableonline (doi:10.1289/ ehp.0901402.S1 via http://dx.doi.org/).

\section{M aterials and M ethods}

\section{Selection criteria}

All epidemiologic studiesincluded in thepreviousIARC M onographswereconsidered (IARC 1989, in press). Further, we searched PubM ed (National Center for Biotechnology Information 2009) for articles in any language describing lung cancer in painters referenced in or published sincetheprevious IARC M onograph (IARC 1989) through 24 August 2009, using the following search terms [by text word (tw), MeSH heading (mh), or publication type (pt)]: "paint*[tw]" or "varnish*[tw]" or "lacquer*[tw]"; and "cancer" or "neoplasms[mh]"; and "casecontrol study[mesh]" or "cohort study[mesh]" or "meta-analysis[mh]" or "review[pt]" or "risk factors[mh]" or "neoplasms/ epidemiology" or "neoplasms/etiology" or "neoplasms/Cl" or "occupational diseases/etiology" or "occupational diseases/epidemiology" or "occupational diseases/Cl" or "occupational diseases/M O" or "occupational exposure/adverse effects" or "death certificates[mh]" or "epidemiologic methods[mh]"; and "lung." Weidentified 121 publications after re- 
stricting results to studies in humans. From the PubM ed search, 69 studies were excluded because they were not epidemiologic studies, did not include original data (they were review articles), did not assess occupation as a painter, or lung cancer was not the outcome. The reference lists of pertinent publications were also reviewed to capture relevant data sources that may not havebeen identified with the search criteria.

The definition of painter varied between studies and often included other occupations exposed to paints such as plasterers, glaziers, wallpaper hangers, artists, decorators, French polishers, and aerographers [seeSupplemental M aterial, Table 4 (doi:10.1289/ehp.0901402.S1) for definitions]. It is likely that paperhangers and other aforementioned occupations work in the same job environment as painters or may also paint; therefore, we considered this category as painters (Carstensen et al. 1988).

To be included in this meta-analysis, studies had to report estimates of the relative risk (RR), odds ratio (OR), standardized incidence ratio (SIR), standardized mortality ratio (SM R), proportionate mortality ratio (PM R), or proportional registration ratio with corresponding $95 \%$ confidence intervals ( $\mathrm{Cls}$ ) for everversusnever occupation as a painter or have provided enough informa-tion that allowed for their computation. For studies that did not report the ever-versusnever painter category, weestimated therisk estimates and $95 \% \mathrm{Cl}$ s for these categories. For studies that reported only point estimates without corresponding $\mathrm{Cls}$, p-values, or standard errors, or did not report the distribution of data to allow for computation of relativerisksand $\mathrm{Cls}$ (also for nonoverlapping populations), we made conservative assumptions to estimate RRs and $95 \%$ $\mathrm{Cls}$ from the data provided on a studybystudy basis. These conservative assumptions underestimated the relative risk (toward the null) and overestimated the width of the $\mathrm{Cl}$ (i.e., by doubling the variance to approximate a $95 \% \mathrm{Cl}$ adjusted for multiple factors).

For example, overlapping lung cancer cases among AfricanAmerican (black) men was identified by M orabia et al. (1992) and M uscat et al. (1998). We accounted for this population overlap by approximating the proportion of black male participants (cases and controls) based on distributions presented in other publications detailing this population, applying this proportion to the distribution presented by Morabia et al. (1992) (for black and whites combined) to determine the number of overlapping subjects, and subtracting the overlapping subjects from the distribution presented in Muscat et al. (1998).

Studies were excluded if estimation was impossible. In Supplemental Material, Tables 1-3 (doi:10.1289/ehp.0901402.S1), weusebracketsto indicate the RRs and $95 \% \mathrm{Cl}$ s we calculated. For studies with over-lapping populations, weincluded only the publication with the most complete study population. Further comments on study quality and any exclusions madeare presented in detail in Supplemental Material, Tables 1-3. In total, weincluded in the meta-analysis 17 cohort and record linkagestudies, 29 case-control studies, and 12 proportionate mortality analyses.

\section{Data abstraction}

All articles were assessed independently by three reviewers (A.A., F.M ., N.K.S.) who extracted data that included authors, publication date, country of origin, characteristics of the study population including sex, and any details on the definition of painters, incidenceversus mortality, lung cancer histology, observed and expected cancer cases (for cohort and proportionate mortality studies), number of exposed cases and controls (for case-control studies), yes/no adjustment for smoking or other occupational carcinogens, relativerisks with corresponding $95 \% \mathrm{Cls}$, and results on exposure-response [see Supplemental Material, Tables1-3 (doi:10.1289/ ehp.0901402.S1)]. If adjusted and unadjusted results were reported, the most valid point estimate (i.e., adjusted for smoking and other variables) was abstracted. Any discrepancies in data collection were resolved by two other reviewers (N.G., K.S.).

Summary statistics calculated

for inclusion in the meta-analysis

For cohort and record linkage studies, relative risk estimates (SIR and SM R) were computed by dividing the observed number of cases by the expected number, based on an external reference population. The corresponding $95 \% \mathrm{Cls}$ were estimated using the PAM COM P program (Taeger et al. 2000). If only subgroup results (e.g., by sex or duration of exposure) were reported, fixed-effects models were used to combine stratum-specific data into onesummary estimate [see Supplemental Material, Tables1 and 2 (doi:10.1289/ehp.0901402.S1)].

Wherever possiblefor the proportionatemortality studies, we used proportional cancer mor- 
tality ratios (calculating expected proportions of cancer deaths based on the proportion of cancer mortality in the referencepopulation) in theanalysis instead of PM Rs as a more conservative approach, because proportional cancer mortality ratios provide a better risk estimate for specific cancer sites when the PM R for all cancer is artificially inflated by a deficit in other causes of death (Dalager et al. 1980) [see Supplemental M aterial, Table 3 (doi:10.1289/ehp.0901402.S1) ]. If several cancer sites are associated with a particular occupation, the PM R can underestimate the RR.

Subgroup analyses were conducted by further restriction to studies with stronger methodologies, such as those studies that adjusted for smoking (Baccarelli et al. 2005; BrüskeH ohlfeld et al. 2000; Burns and Swanson 1991; DeStefani et al. 1996, 2005; Dunn and Weir 1965; $\mathrm{H}$ rubec et al. 1995; Jahn et al. 1999; Kjuus et al. 1986; Lerchen et al. 1987; Levin et al. 1988; $M$ atos et al. 2000; M orabia et al. 1992; M uscat et al. 1998; N otani et al. 1993; Pezzotto and Poletto 1999; Pohlabeln et al. 2000; Pronk et al. 2009; Richiardi et al. 2004; Ronco et al. 1988; Siemiatycki 1991; vanLoon et al. 1997; Viadana et al. 1976; Vineis et al. 1988; Williams et al. 1977; WünschFilho et al. 1998; Zahm et al. 1989; Zeka et al. 2006), other occupa-tional risk factors (Jahn et al. 1999; Ronco et al. 1988; Stockwell and M atanoski 1985; van Loon et al. 1997), or population-based case-control studies that adjusted for smoking (BrüskeHohlfeld et al. 2000; Burns and Swanson 1991; Coggon et al. 1986; J ahn et al. 1999; Lerchen et al. 1987; Levin et al. 1988; Pohlabeln et al. 2000; Richiardi et al. 2004; Ronco et al. 1988; Siemiatycki 1991; Vineis et al. 1988; Zahm et al. 1989; Zeka et al. 2006). Only four of the cohort and record linkage studies provided information on smoking status (Dunn and Weir 1965; H rubec et al. 1995; Pronk et al. 2009; vanLoon et al. 1997).

To allow for inclusion in the meta-analysis, we calculated $95 \% \mathrm{Cl}$ s if they were not presented in the original paper. If a $90 \% \mathrm{Cl}$ was presented and if the upper limit (UL) and lower limit (LL) were proportionally symmetric around the risk ratio (for $R R$ and $O R$; i.e., if $U L / R R=R R / L L$ ), an estimate of the standard error (SE) was calculated by SE = (In UL - InLL/3.29), where $3.29=2 x$ 1.645 for $90 \% \mathrm{Cls}$. If only a $p$ value for the null hypothesis was presented, then a test-based SE was estimated using $S E=(\operatorname{In} R R) / Z p$, whereZp is the value of thestandard-normal test statistic corresponding to the $p$ value using a twotailed test. The $U L$ and $L L$ of the $95 \% \mathrm{Cl}$ were estimated by $R R \pm 1.96$ (SE), where $Z p=1.96$ if $p=0.05$ using a two-tailed test (Rothman et al. 2008). A 95\% Cl corresponding to an unadjusted RR was used in the meta-analysisif a paper did not present enough data to allow for estimation of the adjusted $\mathrm{Cl}$.

\section{Statistical analysis}

Because cancer incidence data are often more accurate than mortality data, weused SIRsin the analyses instead of SM Rs whenever both were presented. However, mortality data for lung cancer are a very reasonable proxy for incidence because of the high fatality of lung cancer and the good quality of data from death certificates (Schottenfeld and Fraumeni 2006). Weperformed a separate meta-analysis for proportionate mortality studies. The PM Rs were, however, not included in the overall meta-analyses because of their often lower quality of exposure assessment and their additional potential for bias. Assuming that the different effect estimates (e.g., SM R, SIR, $R R, O R$ ) represent the relative risk, the data were combined for all of the cohort, record linkage, and case-control studies. Sub-analyses were al so performed by stratifying on study design.

$M$ any of the cohort and record linkage studiesused an external reference population to calculate the expected cases. Theuse of an external reference population may result in a healthy worker effect, so that incidence or mortality rates of cancer in the exposed cohort may spuriously appear lower than in the general population. When the external reference rates used to calculate the expected cases are usually assumed to be known without error, an estimate of the exposure coefficient in aregression could beobtained by a weighted linear regression of the natural log of the adjusted SM R on exposure (Sutton et al. 2000). The risk estimates from nested case-control studies were included with the analysis of cohort studies because, essentially, this design can represent a more efficient way to analyze cohort studies and does not suffer from the problems associated with control selection in a case-control study. Summary ORs (meta-ORs) were obtained separately from the meta-analysis of case-control studies. Subgroup analyses were performed stratified by sex, study region, study design, types of adjustment, and duration of employment.

The I ${ }^{2}$ statistic quantifies the extent of inconsistency among the studies (Higginsand Thompson 2002). $I^{2}$ values of $25-50 \%$ indicate moderate inconsistency, whereas values $>50 \%$ reflect large inconsistencies among studies. We present the $I^{2}$ values instead of the Cochran's Q-statistic 
because the Q -statistic informs about the presence or absence of heterogeneity but does not quantify the extent (Huedo M edina et al. 2006). We used both random and fixed-effect models, with weights equal to the inverse of the variance, to calculate a summary risk estimate (DerSimonian and Laird 1986). Results from random-effects models, which account for heterogeneity among studies, are presented.

We conducted sensitivity analyses by dropping one study at a time and examining its influenceon thesummary effect estimates. Forest plots were used to graphically display the data (Lewis and Clarke 2001). Publication bias was visually assessed using Funnel plots (Deeks et al. 2005). We performed all statistical analyses using STATA (version 10.0; StataCorp, College Station, TX, USA), employing the "metan" command for the meta-analyses (Bradburn 2004).

\section{Results}

We reviewed 74reports published since 1951 assessing the relationship between occupation as a painter and the risk of lung cancer [see Supplemental Material, Tables1-3 (doi:10.1289/ ehp.0901402.S1) ]. The estimates of the relative risk reported in 47 independent studies ranged from 0.60 to 5.76 , with 43 studies reporting an RR > 1.0 (Tables 1 and 2). The combined analysis of 18 cohort and record linkage studies (meta$\mathrm{RR}=1.36 ; 95 \% \mathrm{Cl}, 1.29-1.44 ; \mathrm{I} 2=76.4 \%, \mathrm{p}=0$ ) and 29 case control studies (meta-OR, 1.35; 95\% $\mathrm{Cl}, 1.22-1.51 ; 12=48.4 \%, \mathrm{p}=0.002$ ), including $>$ 11,000 incident cases and/or deaths from lung cancer among painters, demonstrated a significantly increased risk overall in persons who had ever reported occupation as a painter (meta-RR $=1.35 ; 95 \% \mathrm{Cl}, 1.29-1.41 ; 12=63.6 \%, p=0$ ) (Figure 1). Although the results of 13proportionate mortality studies were not included in the combined analysis, they also demonstrated a significantly increased risk of lung cancer in painters (meta-PM R, 1.22; 95\% Cl, 1.17-1.28). The Forest plot (Figurel) shows that there was no obvious trend in risk (at least no obvious trend toward a reduction in risk) over time. An influence analysis showed that dropping individual studies did not significantly alter the results (data not shown).

Relative risks were higher in female paint-ers (meta-RR = 2.04; 95\% Cl, 1.59-2.62) (Jahn et al. 1999; M uscat et al. 1998; O PCS 1958, 1971; Pronk et al. 2009; Pukkala 2009; Zeka et al . 2006) than in males (meta-RR $=1.37 ; 95 \% \mathrm{Cl}, 1.29-1.44$ ). Although there were only seven studies among female painters, the meta-RR was statistically significant. Stratification by study region showed that relative risks were highest in Asia (meta-RR $=1.71 ; 95 \% \mathrm{Cl}, 0.97-3.03 ; \mathrm{I} 2=0 \%, \mathrm{p}=0.86)$, similar in Europe (meta- $\mathrm{RR}=1.3895 \% \mathrm{Cl}, 1.28$ $1.48 ; \mid 2=75.8 \%, p=0$ ) and North America (meta$\mathrm{RR}=1.35 ; 95 \% \mathrm{Cl}, 1.26-1.45 ; \mathrm{I} 2=56.4 \%, \mathrm{p}=$ 0.001 ), and lower in South America (meta- RR = $1.17 ; 95 \% \mathrm{Cl}, 0.77-1.76 ; \mathrm{I} 2=48.8 \%, \mathrm{p}=0.10$ ). Of the few studies that reported results for specific histologies (De Stefani et al. 1996, 2005; Pezzotto and Poletto 1999; Richiardi et al. 2004; Siemiatycki et al. 1987), relative risks were generally highest among those diagnosed with small-cell cancer, although the Cls were wide because of the small number of cases and because results for the different histologic entities were not reported consistently.

There appeared to be no evidence of publication bias among cohort and record linkage studies (data not shown). However, visual inspection of the funnel plot for 30 independent case-control studies demonstrated someevidence of publication bias: the plot was slightly skewed with a deficit of smaller nonpositive studies (represented by large SEs) (Figure 2). When restricting the analysis to the larger case-control studies that showed both positive and negative results, the meta-OR remained significantly elevated (metaOR, 1.31; $95 \% \mathrm{Cl}, 1.18-1.45 ; \mathrm{I} 2=51.6 \%, \mathrm{p}=$ $0.003)$. Therewaslittle differencein the results of case-control studies stratified by hospital-based controls (meta-OR, 1.37; 95\% Cl, 1.09-1.74; I2 = $59.3 \%, p=0.002$ ) or population-based controls (meta-OR, 1.34; 95\% Cl, 1.18-1.51; I $2=25.9 \%$, p $=0.16)$, although the population-based studies were less heterogeneous.

We performed additional analyses to examine the summary estimates when restricted to population-based case-control studies that adjusted for tobacco smoking or other occupational exposures. Restricting to population-based casecontrol studies that adjusted for smoking demonstrated less heterogeneity between studies and strengthened the results (meta-OR, 1.41; 95\% Cl, $\left.1.23-1.61 ;\left.\right|^{2}=0 \%, p=0.45\right)$. Four cohort studies reported smoking-adjusted results (Dunn and Weir 1965; H rubec et al. 1995; Pronk et al. 2009; vanLoon et al. 1997), with a meta RR of 1.22 (95\% $\mathrm{Cl}, 0.97-1.52 ; \mathrm{I}^{2}=23.7 \%, \mathrm{p}=0.27$ ), slightly lower than the meta-RR for cohort studies that did not adjust for smoking (meta-RR $=1.38 ; 95 \% \mathrm{Cl}$, $\left.1.30-1.46 ;\left.\right|^{2}=80.4 \%, p=0\right)$. 
Table 1. Cohort and record linkage studies assessing lung and respiratory cancer among persons with occupation as a painter by publication date.

\begin{tabular}{|c|c|c|c|c|c|c|}
\hline $\begin{array}{l}\text { Reference, } \\
\text { location, and } \\
\text { time period }\end{array}$ & $\begin{array}{c}\text { Cohort } \\
\text { description }\end{array}$ & $\begin{array}{c}\text { Exposure } \\
\text { assessment }\end{array}$ & $\begin{array}{l}\text { Exposure } \\
\text { categories }\end{array}$ & $\begin{array}{l}\text { No. of } \\
\text { cases/ } \\
\text { deaths }\end{array}$ & $\begin{array}{c}\mathrm{HR} / \mathrm{RR} / \mathrm{SI} / \mathrm{R} / \mathrm{SM} \mathrm{R} \\
(95 \% \mathrm{Cl})\end{array}$ & $\begin{array}{l}\text { Adjustment } \\
\text { for potential } \\
\text { confounders }\end{array}$ \\
\hline $\begin{array}{l}\text { Pronk et al. } \\
\text { 2009,Shanghai, } \\
\text { China 1996-2005 }\end{array}$ & $\begin{array}{l}71,067 \text { never- } \\
\text { smoking women } \\
\text { who held a job } \\
\text { outside the home }\end{array}$ & $\begin{array}{l}\text { Detailed lifetime } \\
\text { occupational } \\
\text { histories for each } \\
\text { job held > } 1 \text { year } \\
\text { from in-person } \\
\text { interview }\end{array}$ & $\begin{array}{l}\text { Painter } \\
\text { (construction, } \\
\text { automotive } \\
\text { industry, and } \\
\text { other users) } \\
\text { Years } \\
\text { employment a } \\
<10 \\
\geq 10 \\
<20 \\
\geq 20\end{array}$ & $\begin{array}{l}1 \\
5 \\
5 \\
1\end{array}$ & $\begin{array}{l}0.83(0.12-5.90) \\
2.75(1.12-6.73) \\
2.17(0.89-5.31) \\
1.36(0.19-9.75)\end{array}$ & $\begin{array}{l}\text { Passive } \\
\text { smoking, } \\
\text { family history } \\
\text { of cancer, } \\
\text { education } \\
\text { Age, passive } \\
\text { smoking } \\
\text { (smokers } \\
\text { excluded), } \\
\text { education } \\
\text { level, family } \\
\text { history of lung } \\
\text { cancer }\end{array}$ \\
\hline $\begin{array}{l}\text { Pukkala et al., in } \\
\text { press, Denmark } \\
\text { 1971-2003, } \\
\text { Finland 1971- } \\
\text { 2005, } \\
\text { Iceland1982-2004, } \\
\text { Norway 1961- } \\
\text { 2003, Sweden } \\
1961-2005\end{array}$ & $\begin{array}{l}15 \text { million } \\
\text { people in the } \\
1960,1970 \text {, } \\
\text { 1980/1981, and/ } \\
\text { or } 1990 \text { censuses } \\
\text { and the } 2.8 \\
\text { million incident } \\
\text { cancer cases } \\
\text { diagnosed in } \\
\text { these people in a } \\
\text { follow-up until } \\
\text { about } 2005 \text { were } \\
\text { linked to N ordic } \\
\text { national } \\
\text { registries }\end{array}$ & $\begin{array}{l}\text { Occupation from } \\
\text { self-administered } \\
\text { census } \\
\text { questionnaire }\end{array}$ & $\begin{array}{l}\text { Painters } \\
\text { M en } \\
\text { Women }\end{array}$ & $\begin{array}{c}{[3,465]} \\
3,418 \\
47\end{array}$ & $\begin{array}{c}\text { SIR: }[1.24 \\
(1.20-1.28)] \\
1.23(1.19-1.28) \\
1.90(1.40-2.53)\end{array}$ & $\begin{array}{l}\text { Country, sex, } \\
\text { age, period }\end{array}$ \\
\hline $\begin{array}{l}\text { Boice et al. 1999, } \\
\text { Lockheed Martin } \\
\text { Plant, Burbank, } \\
\text { Los Angeles } \\
\text { County, CA, USA } \\
\text { 1960-1996 }\end{array}$ & $\begin{array}{l}\text { 1,216 painters } \\
\text { ( } 1,139 \text { men, } 77 \\
\text { women) } \\
\text { employed } \geq 1 \\
\text { year in the } \\
\text { aircraft industry }\end{array}$ & $\begin{array}{l}\text { Detailed job history } \\
\text { from work history } \\
\text { cards }\end{array}$ & Painter & 41 & $\begin{array}{c}\text { SM R: } 1.11 \\
{[0.80-1.51]}\end{array}$ & $\begin{array}{l}\text { Age, sex, race, } \\
\text { calendar year }\end{array}$ \\
\hline $\begin{array}{l}\text { Steenland and Palu } \\
\text { 1999, California, } \\
\text { M issouri, N ew } \\
\text { York, Texas, USA, } \\
\text { 1975-1994 }\end{array}$ & $\begin{array}{l}42,170 \text { painters } \\
\text { and } 14,316 \\
\text { nonpainters with } \\
\geq 1 \text { year union } \\
\text { membership }\end{array}$ & $\begin{array}{l}\text { Job titles inferred } \\
\text { from union } \\
\text { membership } \\
\text { records that } \\
\text { identified the } \\
\text { members' specialty } \\
\text { affiliation and trade } \\
\text { of the local union }\end{array}$ & Painter & 1,746 & $\begin{array}{c}\text { SM R: } 1.23 \\
(1.17-1.29)\end{array}$ & $\begin{array}{l}\text { Age, calendar } \\
\text { time }\end{array}$ \\
\hline $\begin{array}{l}\text { van Loon et al. } \\
\text { 1997, The } \\
\text { Netherlands } \\
\text { 1986-1990 }\end{array}$ & $\begin{array}{l}58,729 \text { men, } \\
55-69 \text { years of } \\
\text { age, were } \\
\text { enrolled from } \\
\text { the general } \\
\text { Dutch } \\
\text { population }\end{array}$ & $\begin{array}{l}\text { Paint exposure } \\
\text { from a self- } \\
\text { administered } \\
\text { questionnaire and } \\
\text { case-by-case expert } \\
\text { assessment }\end{array}$ & $\begin{array}{l}\text { Paint dust } \\
\text { exposure } \\
\text { Any }{ }^{b} \\
\text { Low } \\
\text { High } \\
\text { p-Value for } \\
\text { trend }\end{array}$ & $\begin{array}{c}18 \\
4 \\
14\end{array}$ & $\begin{array}{c}\text { RR: }[2.41 \\
(1.07-5.44)] \\
2.29(0.61-8.63) \\
2.48(0.88-6.97) \\
<0.01\end{array}$ & $\begin{array}{l}\text { Age, other } \\
\text { occupational } \\
\text { exposures, } \\
\text { smoking } \\
\text { habits, dietary } \\
\text { intake of } \\
\text { vitamin } C, \beta- \\
\text { carotene, and } \\
\text { retinol }\end{array}$ \\
\hline $\begin{array}{l}\text { Alexander et al. } \\
\text { 1996, Seattle, WA, } \\
\text { USA 1974-1994 }\end{array}$ & $\begin{array}{l}2,429 \text { chromate- } \\
\text { exposed workers } \\
\text { employed } \geq 6 \\
\text { months in the } \\
\text { aerospace } \\
\text { industry }\end{array}$ & $\begin{array}{l}\text { Exposure to } \\
\text { chromium (VI) was } \\
\text { estimated from } \\
\text { industrial hygiene } \\
\text { measurements and } \\
\text { work-history } \\
\text { records }\end{array}$ & All workers & 15 & SIR: $0.8(0.4-1.3)$ & $\begin{array}{l}\text { Age, sex, race, } \\
\text { calendar year }\end{array}$ \\
\hline
\end{tabular}


Table 1. continuation

\begin{tabular}{|c|c|c|c|c|c|c|}
\hline $\begin{array}{l}\text { Reference, } \\
\text { location, and } \\
\text { time period }\end{array}$ & $\begin{array}{c}\text { Cohort } \\
\text { description }\end{array}$ & $\begin{array}{c}\text { Exposure } \\
\text { assessment }\end{array}$ & $\begin{array}{l}\text { Exposure } \\
\text { categories }\end{array}$ & $\begin{array}{l}\text { No. of } \\
\text { cases/ } \\
\text { deaths }\end{array}$ & $\begin{array}{l}\mathrm{HR} / \mathrm{RR} / \mathrm{SIR} / \mathrm{SM} \mathrm{R} \\
(95 \% \mathrm{Cl})\end{array}$ & $\begin{array}{l}\text { Adjustment } \\
\text { for potential } \\
\text { confounders }\end{array}$ \\
\hline $\begin{array}{l}\text { Hrubec et al. 1995, } \\
\text { USA 1954-1980 }\end{array}$ & $\begin{array}{l}\text { 1,178 painters } \\
\text { assembled from a } \\
\text { roster of } \\
\text { approximately } \\
300,000 \text { white } \\
\text { male veterans of } \\
\text { World War I }\end{array}$ & $\begin{array}{l}\text { Occupation and } \\
\text { usual industry of } \\
\text { employment from } \\
\text { mailed } \\
\text { questionnaire }\end{array}$ & $\begin{array}{l}\text { Painters, } \\
\text { construction, } \\
\text { and } \\
\text { maintenance }\end{array}$ & 36 & $\begin{array}{c}\text { SM R: } 1.1 \\
{[0.77-1.43]}\end{array}$ & $\begin{array}{l}\text { Smoking, age, } \\
\text { calendar time }\end{array}$ \\
\hline $\begin{array}{l}\text { Bethune et al. } \\
\text { 1995; OPCS 1995, } \\
\text { England and Wales, } \\
\text { United Kingdom } \\
\text { 1976-1989 }\end{array}$ & $\begin{array}{l}\text { M en from the } \\
1971 \text { and } 1981 \\
\text { census cohorts } \\
\text { who died } \\
\text { between } 1976 \\
\text { and } 1989\end{array}$ & $\begin{array}{l}\text { Occupation from } \\
\text { death certificates }\end{array}$ & $\begin{array}{l}\text { Painters and } \\
\text { decorators }\end{array}$ & NG & $\begin{array}{l}\text { SMR: } 1.51 \\
(1.22-1.85)\end{array}$ & $\begin{array}{l}\text { Age, sex, } \\
\text { calendar year }\end{array}$ \\
\hline $\begin{array}{l}\text { Gubéran et al. } \\
\text { 1989, Switzerland } \\
\text { 1971-1984 }\end{array}$ & $\begin{array}{l}1,916 \text { male } \\
\text { painters from } \\
\text { the } 1970 \text { Geneva } \\
\text { census }\end{array}$ & $\begin{array}{l}\text { Occupation from } \\
\text { the } 1970 \text { census }\end{array}$ & Painters & 40 & $\begin{array}{c}\text { SIR: } 1.47 \\
\text { [1.05-2.00] }\end{array}$ & $\begin{array}{l}\text { Age, sex, } \\
\text { matrimonial } \\
\text { status, calendar } \\
\text { year }\end{array}$ \\
\hline $\begin{array}{l}\text { OPCS 1986, } \\
\text { Scotland, England, } \\
\text { and Wal es, United } \\
\text { Kingdom 1979- } \\
\text { 1980, 1982-1983 }\end{array}$ & $\begin{array}{l}\text { M en in Great } \\
\text { Britain who died } \\
\text { during 1979- } \\
1980 \text { and 1982- } \\
1983 ; \text { mortality } \\
\text { of men 15-74 } \\
\text { years of age in } \\
\text { England and } \\
\text { Wales in } 1981\end{array}$ & $\begin{array}{l}\text { Last full-time } \\
\text { occupation from } \\
\text { death certificate }\end{array}$ & $\begin{array}{l}\text { Painters, } \\
\text { decorators, } \\
\text { French } \\
\text { polishers } \\
\text { Men }\end{array}$ & 779 & $\begin{array}{l}\text { SM R: } 1.44 \\
{[1.34-1.54]}\end{array}$ & Age, sex \\
\hline $\begin{array}{l}\text { Dubrow and } \\
\text { Wegman 1984, } \\
\text { Massachusetts, } \\
\text { USA 1971-1973 }\end{array}$ & $\begin{array}{l}34,879 \text { white } \\
\text { men }>20 \text { years } \\
\text { of age }\end{array}$ & $\begin{array}{l}\text { U sual occupation } \\
\text { from death } \\
\text { certificate }\end{array}$ & $\begin{array}{l}\text { Painters } \\
\text { grouped }\end{array}$ & 110 & $\begin{array}{l}\text { SM R : } 1.31 \\
{[1.08-1.58]}\end{array}$ & Age \\
\hline $\begin{array}{l}\text { Whorton et al. } \\
\text { 1983, San } \\
\text { Francisco/O akland } \\
\text { SM SA, CA, USA } \\
\text { 1976-1978 }\end{array}$ & $\begin{array}{l}2,200 \text { painting } \\
\text { union members } \\
(2,197 \text { men, } 3 \\
\text { women) }\end{array}$ & $\begin{array}{l}\text { 1976-1977 union } \\
\text { membership files }\end{array}$ & Painter & 15 & $\begin{array}{l}\text { SIR: } 1.99 \\
{[1.12-3.30]}\end{array}$ & Age, sex, year \\
\hline $\begin{array}{l}\text { OPCS 1978, } \\
\text { England and Wales, } \\
\text { United Kingdom } \\
\text { 1970-1972 }\end{array}$ & $\begin{array}{l}\text { Registered deaths } \\
\text { of } 273,129 \text { men }\end{array}$ & $\begin{array}{l}\text { Last occupation } \\
\text { recorded on the } \\
\text { death certificate }\end{array}$ & $\begin{array}{l}\text { Painters and } \\
\text { decorators }\end{array}$ & 847 & $\begin{array}{c}\text { SMR: } 1.39 \\
{[1.30-1.49]}\end{array}$ & Age, sex \\
\hline $\begin{array}{l}\text { M enck and } \\
\text { Henderson 1976, } \\
\text { Los Angeles } \\
\text { County, CA, USA } \\
\text { 1968-1970 }\end{array}$ & $\begin{array}{l}\text { Pooled mortality } \\
\text { and morbidity } \\
\text { data of } 2,161 \\
\text { deaths from lung } \\
\text { cancer and } 1,777 \\
\text { incident cases of } \\
\text { lung cancer } \\
\text { among white } \\
\text { males }\end{array}$ & $\begin{array}{l}\text { Last occupation } \\
\text { from death } \\
\text { certificates and } \\
\text { surveillance registry } \\
\text { files }\end{array}$ & Painter & 87 & $\begin{array}{l}\text { SMR: } 1.58 \\
{[1.27-1.95]}\end{array}$ & Age \\
\hline $\begin{array}{l}\text { OPCS 1971, } \\
\text { England and Wales, } \\
\text { United Kingdom } \\
\text { 1959-1963 }\end{array}$ & $\begin{array}{l}\text { Registered deaths } \\
\text { of men and } \\
\text { women in } \\
\text { England and } \\
\text { Wales }\end{array}$ & $\begin{array}{l}\text { Last occupation } \\
\text { from death } \\
\text { certificate }\end{array}$ & $\begin{array}{l}\text { Painters and } \\
\text { decorators } \\
15-64 \text { years } \\
\text { of age } \\
\text { Men and } \\
\text { women } \\
\text { Men } \\
\text { Single } \\
\text { women }\end{array}$ & $\begin{array}{c}1,506 \\
1,502 \\
4\end{array}$ & $\begin{array}{c}\text { SMR: } 1.43 \\
{[1.36-1.51]} \\
1.43[1.36-1.50] \\
4.00[1.09-10.24]\end{array}$ & Age, sex \\
\hline
\end{tabular}


Table 1. continuation

\begin{tabular}{|c|c|c|c|c|c|c|}
\hline $\begin{array}{l}\text { Reference, } \\
\text { location, and } \\
\text { time period }\end{array}$ & $\begin{array}{c}\text { Cohort } \\
\text { description }\end{array}$ & $\begin{array}{l}\text { Exposure } \\
\text { assessment }\end{array}$ & $\begin{array}{l}\text { Exposure } \\
\text { categories }\end{array}$ & $\begin{array}{l}\text { No. of } \\
\text { cases/ } \\
\text { deaths }\end{array}$ & $\begin{array}{l}\mathrm{HR} / \mathrm{RR} / \mathrm{SI} / \mathrm{R} / \mathrm{SMR} \\
(95 \% \mathrm{CI})\end{array}$ & $\begin{array}{l}\text { Adjustment } \\
\text { for potential } \\
\text { confounders }\end{array}$ \\
\hline $\begin{array}{l}\text { Dunn and Weir } \\
\text { 1965, California, } \\
\text { USA 1954-1962 }\end{array}$ & $\begin{array}{l}\text { Prospective study } \\
\text { of > } 68,000 \text { men } \\
\text { working in } \\
\text { "suspicious" } \\
\text { occupations } \\
\text { ( } 12,512 \text { painters } \\
\text { and decorators) }\end{array}$ & $\begin{array}{l}\text { M en were enrolled } \\
\text { based on their } \\
\text { occupation, } \\
\text { identified through } \\
\text { unions, and mailed } \\
\text { questionnaire }\end{array}$ & $\begin{array}{l}\text { Painters and } \\
\text { decorators }\end{array}$ & 91 & $\begin{array}{l}\text { SM R: } 1.14 \\
{[0.92-1.40]}\end{array}$ & Age, smoking \\
\hline $\begin{array}{l}\text { Enterline and } \\
\text { M cKiever } 1963 \\
\text { Guralnick 1963, } \\
\text { USA } 1950\end{array}$ & $\begin{array}{l}\text { Men who died in } \\
\text { the USA in } 1950\end{array}$ & $\begin{array}{l}\text { Usual occupation } \\
\text { and industry } \\
\text { recorded from } \\
\text { death certificates }\end{array}$ & $\begin{array}{l}\text { Painters ans } \\
\text { plasterers }\end{array}$ & 118 & $\begin{array}{c}\text { SM R: } 1.51 \\
{[1.25-1.81]}\end{array}$ & Age, race \\
\hline $\begin{array}{l}\text { OPCS 1958, } \\
\text { England and Wales, } \\
\text { United Kingdom } \\
\text { 1949-1953 }\end{array}$ & $\begin{array}{l}\text { Registered deaths } \\
\text { of } 221,941 \text { men } \\
\text { and women in } \\
\text { the broad } \\
\text { occupational } \\
\text { category of } \\
\text { painters and } \\
\text { decorators }\end{array}$ & $\begin{array}{l}\text { Occupation at time } \\
\text { of death or last } \\
\text { occupation from } \\
\text { death certificates }\end{array}$ & $\begin{array}{l}\text { Other } \\
\text { painters and } \\
\text { decorators } \\
\text { M en and } \\
\text { women } \\
\text { M en } \\
\text { Single } \\
\text { women }\end{array}$ & $\begin{array}{c}912 \\
909 \\
3\end{array}$ & $\begin{array}{c}\text { SM R: }[1.49 \\
(1.40-1.59)] \\
{[1.49(1.40-1.59)]} \\
3.00[0.62-8.77]\end{array}$ & Age, sex \\
\hline
\end{tabular}

Abbreviations: HR, hazard ratio; NG, not given; SM SA, standard metropolitan statistical area. Values in brackets were calculated

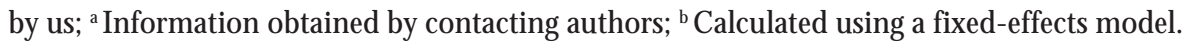

Table 2. Case-control studies of the association between lung cancer and occupation as a painter by publication date.

\begin{tabular}{|c|c|c|c|c|c|c|c|}
\hline $\begin{array}{l}\text { Reference, } \\
\text { location, and } \\
\text { time period }\end{array}$ & $\begin{array}{c}\text { Characteristics } \\
\text { of cases }\end{array}$ & $\begin{array}{c}\text { Characteristics } \\
\text { of controls }\end{array}$ & $\begin{array}{c}\text { Exposure } \\
\text { assessment }\end{array}$ & Exposure & $\begin{array}{l}\text { No. of } \\
\text { exposed } \\
\text { cases }\end{array}$ & OR $(95 \% \mathrm{Cl})$ & $\begin{array}{l}\text { Adjustment } \\
\text { for potential } \\
\text { confounders }\end{array}$ \\
\hline $\begin{array}{l}\text { Zeka et al. 2006, } \\
\text { Czech Republic, } \\
\text { Hungary, Poland, } \\
\text { Romania, Russia, } \\
\text { Slovakia, United } \\
\text { Kingdom } \\
\text { 1998- } 2002\end{array}$ & $\begin{array}{l}223 \text { never- } \\
\text { smoking cases } \\
\text { ( } 48 \text { men, } 175 \\
\text { women) }\end{array}$ & $\begin{array}{l}1,039 \\
\text { nonsmoking } \\
\text { controls ( } 534 \\
\text { men, } 505 \\
\text { women) }\end{array}$ & $\begin{array}{l}\text { Lifetime } \\
\text { occupational } \\
\text { histories for } \\
\text { jobs held } \geq 1 \\
\text { year from } \\
\text { in-person } \\
\text { interview }\end{array}$ & $\begin{array}{l}\text { Painters } \\
\text { Man and } \\
\text { woman } \\
\text { Woman }\end{array}$ & $\begin{array}{l}6 \\
6\end{array}$ & $\begin{array}{c}{[1.81} \\
(0.72-4.59)] \\
1.8(0.53-6.0)\end{array}$ & $\begin{array}{l}\text { None } \\
\text { Sex, age, } \\
\text { study center }\end{array}$ \\
\hline $\begin{array}{l}\text { Baccarelli et al. } \\
\text { 2005, Leningrad } \\
\text { Province } \\
\text { (Russia) } \\
\text { 1993-1998 }\end{array}$ & $\begin{array}{l}540(474 \\
\text { men, } 66 \\
\text { women }\end{array}$ & $\begin{array}{l}582 \text { (453 men, } \\
129 \text { women) } \\
\text { individuals } \\
\text { withautopsy- } \\
\text { based } \\
\text { diagnoses of } \\
\text { non-cancer- } \\
\text { related and } \\
\text { non-tobacco- } \\
\text { related } \\
\text { conditions, } \\
\text { frequency } \\
\text { matched by } \\
\text { sex, age, area, } \\
\text { year of death }\end{array}$ & $\begin{array}{l}\text { Lifetime } \\
\text { occupational } \\
\text { histories } \\
\text { from } \\
\text { personal } \\
\text { records }\end{array}$ & $\begin{array}{l}\text { Ever } \\
\text { painters } \\
<10 \text { years } \\
\geq 10 \text { years }\end{array}$ & $\begin{array}{l}10 \\
6 \\
4\end{array}$ & $\begin{array}{l}0.6(0.3-1.4) \\
0.5(0.2-1.5) \\
0.8(0.2-3.0)\end{array}$ & $\begin{array}{l}\text { Age, sex, } \\
\text { smoking }\end{array}$ \\
\hline
\end{tabular}


Table 2. continuation

\begin{tabular}{|c|c|c|c|c|c|c|c|}
\hline $\begin{array}{l}\text { Reference, } \\
\text { location, and } \\
\text { time period }\end{array}$ & $\begin{array}{c}\text { Characteristics } \\
\text { of cases }\end{array}$ & $\begin{array}{c}\text { Characteristics } \\
\text { of controls }\end{array}$ & $\begin{array}{c}\text { Exposure } \\
\text { assessment }\end{array}$ & Exposure & $\begin{array}{l}\text { No. of } \\
\text { exposed } \\
\text { cases }\end{array}$ & OR $(95 \% \mathrm{Cl})$ & $\begin{array}{l}\text { Adjustment } \\
\text { for potential } \\
\text { confounders }\end{array}$ \\
\hline $\begin{array}{l}\text { De Stefani et al. } \\
2005 \text {, } \\
\text { M ontevideo, } \\
\text { Uruguay } \\
\text { 1994- } 2000\end{array}$ & 338 men & $\begin{array}{l}1,014 \text { males } \\
\text { hospitalized } \\
\text { for conditions } \\
\text { not related to } \\
\text { tobacco } \\
\text { smoking, } \\
\text { matched by } \\
\text { age, residence } \\
\text { and urban/ } \\
\text { rural status }\end{array}$ & $\begin{array}{l}\text { Lifetime } \\
\text { occupational } \\
\text { history from } \\
\text { in-person } \\
\text { interview }\end{array}$ & $\begin{array}{l}\text { Ever painter } \\
\text { Employment } \\
\text { (years) } \\
1-20 \\
\geq 21 \\
\text { p for trend }\end{array}$ & 26 & $\begin{array}{c}1.8(1.0-3.1) \\
\\
9.6(2.6-36.0) \\
1.2(0.6-2.2) \\
0.07\end{array}$ & $\begin{array}{l}\text { Age, } \\
\text { residence, } \\
\text { urban/rural } \\
\text { status, } \\
\text { education, } \\
\text { smoking } \\
\text { status and } \\
\text { years since } \\
\text { quitting and } \\
\text { age at start, } \\
\text { no. of } \\
\text { cigarettes per } \\
\text { day }\end{array}$ \\
\hline $\begin{array}{l}\text { Richiardi et al. } \\
\text { 2004, Turin and } \\
\text { Easten Veneto, } \\
\text { Italy, 1990-1992 }\end{array}$ & 956 men & $\begin{array}{l}1,253 \text { male } \\
\text { population- } \\
\text { based controls, } \\
\text { matched by } \\
\text { study area, 5- } \\
\text { year age groups }\end{array}$ & $\begin{array}{l}\text { Lifetime } \\
\text { occupational } \\
\text { history from } \\
\text { in-person } \\
\text { interview }\end{array}$ & $\begin{array}{l}\text { Ever } \\
\text { painters }\end{array}$ & 62 & $1.7(1.1-2.8)$ & $\begin{array}{l}\text { Age, study } \\
\text { area, } \\
\text { smoking } \\
\text { (never, ex-, } \\
\text { active } \\
\text { smokers), } \\
\text { no. of job } \\
\text { periods, } \\
\text { education }\end{array}$ \\
\hline $\begin{array}{l}\text { Bouchardy et al. } \\
\text { 2002, cantons of } \\
\text { Basel, Geneva, St } \\
\text { Gall, Vaud, and } \\
\text { Zurich, } \\
\text { Switzerland, } \\
\text { 1980-1993 }\end{array}$ & 9,106 men & $\begin{array}{l}49,028 \text { male } \\
\text { non-lung } \\
\text { cancer } \\
\text { registrants }\end{array}$ & $\begin{array}{l}\text { Longest, } \\
\text { current, or } \\
\text { most recent } \\
\text { occupation } \\
\text { as recorded } \\
\text { at the time } \\
\text { of } \\
\text { registration } \\
\text { (main or } \\
\text { best- } \\
\text { specified } \\
\text { occupation } \\
\text { in Zurich } \\
\text { Registry) }\end{array}$ & $\begin{array}{l}\text { Plasterers } \\
\text { and painters } \\
\text { (in the } \\
\text { construction } \\
\text { industry) }\end{array}$ & 273 & $1.1(1.0-1.3)$ & $\begin{array}{l}\text { Age, registry, } \\
\text { civil status, } \\
\text { period of } \\
\text { diagnosis, } \\
\text { nationality, } \\
\text { urban/rural } \\
\text { residence, } \\
\text { socioeconomic } \\
\text { status, } \\
\text { histologic } \\
\text { confirmation, } \\
\text { information } \\
\text { from death } \\
\text { certificate } \\
\text { only (cases) }\end{array}$ \\
\hline $\begin{array}{l}\text { Matos et al. } \\
\text { 2000, Buenos } \\
\text { Aires, Argentina, } \\
\text { 1994- } 1996\end{array}$ & 200 men & $\begin{array}{l}397 \text { male } \\
\text { controls } \\
\text { hospitalized } \\
\text { for non- } \\
\text { tobacco- } \\
\text { related } \\
\text { conditions, } \\
\text { matched by } \\
\text { hospital and } \\
\text { age }\end{array}$ & $\begin{array}{l}\text { Full } \\
\text { occupational } \\
\text { history from } \\
\text { in-person } \\
\text { interview. } \\
\text { Further } \\
\text { details } \\
\text { requested } \\
\text { for } \\
\text { occupations } \\
\text { held }>1 \text { year }\end{array}$ & $\begin{array}{l}\text { Ever } \\
\text { painters }\end{array}$ & 16 & $1.2(0.5-2.4)$ & $\begin{array}{l}\text { Age, } \\
\text { hospital, } \\
\text { smoking } \\
\text { (pack-years), } \\
\text { other } \\
\text { occupations } \\
\text { with } \\
\text { significant } \\
\text { ORs } \\
\text { ( } p<0.05 \text { ) }\end{array}$ \\
\hline $\begin{array}{l}\text { Pohlabeln et al. } \\
2000,12 \text { centers } \\
\text { in Germany, } \\
\text { Italy, Portugal, } \\
\text { Sweden, U nited } \\
\text { Kingdom, } \\
\text { France, and } \\
\text { Spain, } \\
\text { 1988-1994 }\end{array}$ & $\begin{array}{l}650 \\
\text { nonsmoking } \\
\text { cases a }(509 \\
\text { women, } 141 \\
\text { men) }\end{array}$ & $\begin{array}{l}1,542 \\
\text { nonsmoking } \\
\text { controls } \\
\text { ( } 1,011 \\
\text { females, } 531 \\
\text { males) }\end{array}$ & $\begin{array}{l}\text { In-person } \\
\text { interview for } \\
\text { lifetime } \\
\text { occupational } \\
\text { history }\end{array}$ & $\begin{array}{l}\text { Ever } \\
\text { painters } \\
\text { (men) }\end{array}$ & 6 & $1.84(0.59-5.74)$ & Age, center \\
\hline
\end{tabular}


Table 2. continuation

\begin{tabular}{|c|c|c|c|c|c|c|c|}
\hline $\begin{array}{l}\text { Reference, } \\
\text { location, and } \\
\text { time period }\end{array}$ & $\begin{array}{c}\text { Characteristics } \\
\text { of cases }\end{array}$ & $\begin{array}{c}\text { Characteristics } \\
\text { of controls }\end{array}$ & $\begin{array}{l}\text { Exposure } \\
\text { assessment }\end{array}$ & Exposure & $\begin{array}{l}\text { No. of } \\
\text { exposed } \\
\text { cases }\end{array}$ & OR $(95 \% \mathrm{Cl})$ & $\begin{array}{l}\text { Adjustment } \\
\text { for potential } \\
\text { confounders }\end{array}$ \\
\hline $\begin{array}{l}\text { Jahn et al. 1999; } \\
\text { Bruske-H ohlfeld } \\
\text { et al. 2000 b, } \\
\text { Germany, } \\
\text { 1988-1993, } \\
\text { 1990-1996 }\end{array}$ & $\begin{array}{l}686 \text { women, } \\
3,498 \text { men }\end{array}$ & $\begin{array}{l}712 \text { female } \\
\text { and } 3,541 \\
\text { male } \\
\text { population } \\
\text { controls }\end{array}$ & $\begin{array}{l}\text { Full } \\
\text { occupational } \\
\text { history and } \\
\text { supplementary } \\
\text { job-specific } \\
\text { modules } \\
\text { from in- } \\
\text { person } \\
\text { interview }\end{array}$ & $\begin{array}{l}\text { Ever } \\
\text { painters } \\
\text { (women) } \\
\text { Ever } \\
\text { painters/ } \\
\text { lacquerers } \\
\text { M en } \\
\text { Men and } \\
\text { women }\end{array}$ & $\begin{array}{c}147 \\
{[160]}\end{array}$ & $\begin{array}{c}3.0(0.73-12.33) \\
\\
\\
\\
\\
1.42(1.05-1.92) \\
{[1.47} \\
(1.09-1.97)]^{c}\end{array}$ & $\begin{array}{l}\text { Smoking, } \\
\text { asbestos, } \\
\text { education, } \\
\text { age, region } \\
\text { of residence }\end{array}$ \\
\hline $\begin{array}{l}\text { Pezzotto and } \\
\text { Poletto 1999, } \\
\text { Rosario City, } \\
\text { Argentina, } \\
\text { 1992-1998 }\end{array}$ & 367 men & $\begin{array}{l}586 \text { hospital- } \\
\text { based males } \\
\text { controls } \\
\text { admitted for a } \\
\text { non-smoking- } \\
\text { related disease } \\
\text { at the same } \\
\text { hospitals for } \\
\text { traumatic } \\
\text { conditions, } \\
\text { urologic } \\
\text { diseases, acute } \\
\text { surgical } \\
\text { conditions, } \\
\text { and other } \\
\text { illnesses, } \\
\text { matched by } \\
\text { age ( } \pm 3 \text { years); } \\
\text { mean age } 60.1 \\
\pm 10.2 \text { years }\end{array}$ & $\begin{array}{l}\text { Lifetime } \\
\text { occupational } \\
\text { history for } \\
\text { each job } \\
\text { held > } 1 \text { year } \\
\text { from } \\
\text { standardized } \\
\text { questionnaire }\end{array}$ & $\begin{array}{l}\text { House } \\
\text { painters }\end{array}$ & 4 & $2.4(0.4-19.4)$ & $\begin{array}{l}\text { Age, } \\
\text { smoking } \\
\text { habit, } \\
\text { lifelong } \\
\text { cigarette } \\
\text { consumption }\end{array}$ \\
\hline $\begin{array}{l}\text { Muscat et al. } \\
\text { 1998, N ew York } \\
\text { City, Long } \\
\text { Island, NY; } \\
\text { Philadelphia, PA; } \\
\text { Washington, } \\
\text { DC; Detroit, MI; } \\
\text { Chicago, IL, } \\
\text { USA, } \\
\text { 1978-1996 }\end{array}$ & $\begin{array}{l}365 \text { black } \\
\text { men and } 185 \\
\text { black women }\end{array}$ & $\begin{array}{l}251 \text { male and } \\
135 \text { female } \\
\text { black patients; } \\
\text { conditions } \\
\text { unrelated to } \\
\text { tobacco use, } \\
\text { matched by } \\
\text { race, sex, 5- } \\
\text { year age } \\
\text { groups, month } \\
\text { of diagnosis }\end{array}$ & $\begin{array}{l}\text { Only “usual” } \\
\text { occupation } \\
\text { and whether } \\
\text { the job } \\
\text { entailed } \\
\text { regular } \\
\text { exposure to } \\
\text { an } \\
\text { occupational } \\
\text { exposure } \\
\text { (for a } \\
\text { minimum } \\
\text { of } 8 \text { hr/ } \\
\text { week) was } \\
\text { obtained } \\
\text { from } \\
\text { interviews } \\
\text { with subjects } \\
\text { or their next } \\
\text { of kin or } \\
\text { death } \\
\text { certificates }\end{array}$ & $\begin{array}{l}\text { Ever } \\
\text { painters } \\
\text { M end } \\
\text { Women }\end{array}$ & $\begin{array}{c}{[24]} \\
{[19]} \\
5\end{array}$ & $\begin{array}{c}{[1.32} \\
(1.30-1.35)]^{c} \\
{[0.68} \\
(0.29-1.59)] \\
1.8(0.3-12.3)\end{array}$ & $\begin{array}{l}\text { Age, } \\
\text { education, } \\
\text { smoking }\end{array}$ \\
\hline $\begin{array}{l}\text { Wünsch-Filho et } \\
\text { al. 1998, São } \\
\text { Paulo, Brazil, } \\
\text { 1990-1991 }\end{array}$ & $\begin{array}{l}398 \text { cases ( } 307 \\
\text { men, } 91 \\
\text { women) }\end{array}$ & $\begin{array}{l}860 \text { controls } \\
\text { ( } 546 \text { men, } 314 \\
\text { women) } \\
\text { hospitalized } \\
\text { for non- } \\
\text { tobacco- } \\
\text { related } \\
\text { conditions, } \\
\text { matched by } \\
\text { age, sex, } \\
\text { hospital }\end{array}$ & $\begin{array}{l}\text { Full } \\
\text { occupational } \\
\text { history from } \\
\text { in-person } \\
\text { interview }\end{array}$ & $\begin{array}{l}\text { Ever } \\
\text { painters } \\
\text { (men) }\end{array}$ & 128 & $0.77(0.56-1.08)$ & $\begin{array}{l}\text { Age, sex, } \\
\text { hospital, } \\
\text { smoking, } \\
\text { cancer in } \\
\text { family, } \\
\text { migration } \\
\text { history, } \\
\text { socioeconomic } \\
\text { status }\end{array}$ \\
\hline
\end{tabular}

it continues 
Table 2. continuation

\begin{tabular}{|c|c|c|c|c|c|c|c|}
\hline $\begin{array}{l}\text { Reference, } \\
\text { location, and } \\
\text { time period }\end{array}$ & $\begin{array}{c}\text { Characteristics } \\
\text { of cases }\end{array}$ & $\begin{array}{l}\text { Characteristics } \\
\text { of controls }\end{array}$ & $\begin{array}{l}\text { Exposure } \\
\text { assessment }\end{array}$ & Exposure & $\begin{array}{l}\text { No. of } \\
\text { exposed } \\
\text { cases }\end{array}$ & OR $(95 \% \mathrm{Cl})$ & $\begin{array}{l}\text { Adjustment } \\
\text { for potential } \\
\text { confounders }\end{array}$ \\
\hline $\begin{array}{l}\text { De Stefani et al. } \\
\text { 1996, } \\
\text { M ontevideo, } \\
\text { Uruguay, } \\
\text { 1993-1994, } \\
\text { South America }\end{array}$ & 270 men & $\begin{array}{l}383 \text { male } \\
\text { hospital-based } \\
\text { controls: other } \\
\text { cancer sites } \\
\text { except oral } \\
\text { cavity, pharynx, } \\
\text { esophagus, } \\
\text { stomach, } \\
\text { larynx, and } \\
\text { bladder }\end{array}$ & $\begin{array}{l}\text { Lifetime } \\
\text { occupational } \\
\text { history from } \\
\text { in-person } \\
\text { interview }\end{array}$ & $\begin{array}{l}\text { Ever } \\
\text { painters } \\
\text { Employment } \\
\text { (years) } \\
1-20 \\
\geq 21\end{array}$ & 18 & $\begin{array}{l}1.2(0.6-2.4) \\
0.9(0.2-3.0) \\
1.4(0.6-3.1)\end{array}$ & $\begin{array}{l}\text { Age, } \\
\text { residence, } \\
\text { education, } \\
\text { tobacco } \\
\text { smoking } \\
\text { (pack-years), } \\
\text { alcohol } \\
\text { consumption }\end{array}$ \\
\hline $\begin{array}{l}\text { Finkelstein } 1995, \\
\text { Hamilton and } \\
\text { Sault Ste-M arie, } \\
\text { Ontario, Canada, } \\
\text { 1979-1988 }\end{array}$ & 967 men & $\begin{array}{l}2,821 \text { men who } \\
\text { died of ay cause } \\
\text { other than lung } \\
\text { cancer, matched } \\
\text { by age, year of } \\
\text { death, and city } \\
\text { of residence }\end{array}$ & $\begin{array}{l}\text { Occupation } \\
\text { (job and } \\
\text { industry) } \\
\text { from death } \\
\text { certificate }\end{array}$ & $\begin{array}{l}\text { Painters and } \\
\text { plasterers }\end{array}$ & 16 & $1.25(0.63-2.36)$ & $\begin{array}{l}\text { Age, year of } \\
\text { death, city of } \\
\text { residence }\end{array}$ \\
\hline $\begin{array}{l}\text { Notani et al. } \\
1993 \\
\text { Bombay, India, } \\
\text { 1986-1990 }\end{array}$ & 246 men & $\begin{array}{l}212 \text { male } \\
\text { hospital-based } \\
\text { controls } \\
\text { diagnosed with } \\
\text { cancers of the } \\
\text { mouth and oro } \\
\text { - or } \\
\text { hypopharynx } \\
\text { and } \\
\text { noncancerous } \\
\text { oral disease, } \\
\text { frequency } \\
\text { matched by age } \\
\text { and community }\end{array}$ & $\begin{array}{l}\text { Lifetime } \\
\text { occupational } \\
\text { history from } \\
\text { in-person } \\
\text { interview }\end{array}$ & $\begin{array}{l}\text { Ever } \\
\text { painters }\end{array}$ & 6 & $1.62(0.4-7.0)$ & $\begin{array}{l}\text { Age, } \\
\text { community, } \\
\text { smoking } \\
\text { (two groups) }\end{array}$ \\
\hline $\begin{array}{l}\text { Swanson et al. } \\
\text { 1993, Detroit, } \\
\text { M I, metropolitan } \\
\text { area, USA, 1984- } \\
1987\end{array}$ & $\begin{array}{l}3,792 \text { males } \\
(2,866 \text { white, } \\
926 \text { black })\end{array}$ & $\begin{array}{l}1,966 \text { males } \\
\text { (1,596 white, } \\
370 \text { black) with } \\
\text { colon and rectal } \\
\text { cancer }\end{array}$ & $\begin{array}{l}\text { Lifetime } \\
\text { occupational } \\
\text { and } \\
\text { smoking } \\
\text { history from } \\
\text { telephone } \\
\text { interviews } \\
\text { with subjects } \\
\text { or their } \\
\text { surrogates }\end{array}$ & $\begin{array}{l}\text { Painting } \\
\text { machine } \\
\text { operators, } \\
\text { black and } \\
\text { white } \\
<10 \text { years } \\
\geq 10 \text { years } \\
<20 \text { years } \\
\geq 20 \text { years }\end{array}$ & $\begin{array}{l}40 \\
40 \\
53 \\
27\end{array}$ & $\begin{array}{c}{[1.19} \\
(0.61-2.34)]^{\mathrm{e}} \\
{[2.23} \\
(1.05-4.73)]^{\mathrm{e}} \\
{[1.15} \\
(0.65-2.04)]^{\mathrm{e}} \\
{[4.62} \\
(1.61-13.31)]^{\mathrm{e}}\end{array}$ & $\begin{array}{l}\text { Age at } \\
\text { diagnosis, } \\
\text { pack-years of } \\
\text { cigarette } \\
\text { smoking }\end{array}$ \\
\hline $\begin{array}{l}\text { M orabia et al. } \\
\text { 1992, D etroit, } \\
\text { MI; Chicago, IL; } \\
\text { Philadelphia, PA; } \\
\text { Pittsburgh, PA; } \\
\text { New York, NY; } \\
\text { Long Island, NY; } \\
\text { San Francisco, } \\
\text { CA; Birmingham, } \\
\text { AL, USA, } \\
\text { 1980-1989, } \\
\text { American H ealth } \\
\text { Foundation study }\end{array}$ & 1,793 men & $\begin{array}{l}3,228 \text { controls } \\
\text { not hospitalized } \\
\text { for lung cancer } \\
\text { but including } \\
\text { tobacco-related } \\
\text { conditions; } \\
\text { matched by age, } \\
\text { race, hospital, } \\
\text { smoking } \\
\text { history, } \\
\text { admission date }\end{array}$ & $\begin{array}{l}\text { "Usual" } \\
\text { occupation } \\
\text { and } \\
\text { exposure } \\
\text { circumstances } \\
\text { from in- } \\
\text { person } \\
\text { interview }\end{array}$ & Painters & [13] & $0.8(0.32-2.03)$ & $\begin{array}{l}\text { Age, } \\
\text { geographic } \\
\text { area, race, } \\
\text { smoking, } \\
\text { study period }\end{array}$ \\
\hline
\end{tabular}


Table 2. continuation

\begin{tabular}{|c|c|c|c|c|c|c|c|}
\hline $\begin{array}{l}\text { Reference, } \\
\text { location, and } \\
\text { time period }\end{array}$ & $\begin{array}{c}\text { Characteristics } \\
\text { of cases }\end{array}$ & $\begin{array}{l}\text { Characteristics } \\
\text { of controls }\end{array}$ & $\begin{array}{c}\text { Exposure } \\
\text { assessment }\end{array}$ & Exposure & $\begin{array}{l}\text { No. of } \\
\text { exposed } \\
\text { cases }\end{array}$ & OR $(95 \% \mathrm{Cl})$ & $\begin{array}{l}\text { Adjustment } \\
\text { for potential } \\
\text { confounders }\end{array}$ \\
\hline $\begin{array}{l}\text { Burns and } \\
\text { Swanson 1991, } \\
\text { Detroit, MI, } \\
\text { metropolitan } \\
\text { area, USA }\end{array}$ & $\begin{array}{l}5,935(3,918 \\
\text { males, 2,017 } \\
\text { females) }\end{array}$ & $\begin{array}{l}3,956 \text { (1,981 } \\
\text { males, 1,975 } \\
\text { females) with } \\
\text { colon and } \\
\text { rectal cancer }\end{array}$ & $\begin{array}{l}\text { Lifetime } \\
\text { occupational } \\
\text { history from } \\
\text { tel ephone } \\
\text { interviews to } \\
\text { the subjects } \\
\text { or to their } \\
\text { surrogates }\end{array}$ & $\begin{array}{l}\text { Painters } \\
\text { (usual } \\
\text { occupation, } \\
\text { grouped) }\end{array}$ & 97 & $1.96(1.23-3.13)$ & $\begin{array}{l}\text { Age at } \\
\text { diagnosis, } \\
\text { race, } \\
\text { smoking, sex }\end{array}$ \\
\hline $\begin{array}{l}\text { Siemiatycki } \\
\text { 1991, M ontreal, } \\
\text { Canada, } \\
\text { 1979-1985 }\end{array}$ & 857 men & $\begin{array}{l}533 \\
\text { population } \\
\text { controls, } \\
1,360 \text { cancer } \\
\text { controls }\end{array}$ & $\begin{array}{l}\text { Lifetime } \\
\text { occupational } \\
\text { history from } \\
\text { interview }\end{array}$ & $\begin{array}{l}\text { Construction } \\
\text { painter } \\
\text { Any } \\
\text { exposure }\end{array}$ & 26 & $1.4(0.77-2.17)$ & $\begin{array}{l}\text { Age, family } \\
\text { income, } \\
\text { ethnicity, } \\
\text { respondent } \\
\text { type, } \\
\text { cigarette and } \\
\text { alcohol } \\
\text { index }\end{array}$ \\
\hline $\begin{array}{l}\text { Bethwaite et al. } \\
\text { 1990, New } \\
\text { Zealand, } \\
\text { 1980-1984 }\end{array}$ & 4,224 men & $\begin{array}{l}15,680 \text { male } \\
\text { non-lung } \\
\text { cancer } \\
\text { registrants }\end{array}$ & $\begin{array}{l}\text { Current/ } \\
\text { most recent } \\
\text { occupation } \\
\text { as recorded } \\
\text { at the time } \\
\text { of } \\
\text { registration; } \\
\text { smoking } \\
\text { history } \\
\text { obtained } \\
\text { through } \\
\text { tel ephone } \\
\text { interview }\end{array}$ & $\begin{array}{l}\text { Painter } \\
\text { decorators, } \\
\text { steel and } \\
\text { other } \\
\text { construction } \\
\text { painters, car } \\
\text { painters, } \\
\text { spray } \\
\text { painters, } \\
\text { signwriters, } \\
\text { other } \\
\text { unclassified } \\
\text { painters }\end{array}$ & 88 & $1.12(0.93-1.52)$ & Age \\
\hline $\begin{array}{l}\text { Zahm et al. } \\
\text { 1989, M issouri, } \\
\text { USA, } \\
\text { 1980- } 1985\end{array}$ & $\begin{array}{l}\text { 4,431 white } \\
\text { male cases }\end{array}$ & $\begin{array}{l}11,326 \text { white } \\
\text { male non- } \\
\text { lung cancer } \\
\text { registrants }\end{array}$ & $\begin{array}{l}\text { Occupation } \\
\text { at the time } \\
\text { of diagnosis } \\
\text { abstracted } \\
\text { from } \\
\text { medical } \\
\text { records }\end{array}$ & $\begin{array}{l}\text { Painters, } \\
\text { paper } \\
\text { hangers, } \\
\text { plasterers }\end{array}$ & 37 & $2.0(1.2-3.3)$ & $\begin{array}{l}\text { Age, } \\
\text { smoking }\end{array}$ \\
\hline
\end{tabular}

\begin{tabular}{|c|c|c|c|c|c|c|c|}
\hline $\begin{array}{l}\text { Levin et al. } \\
\text { 1988, China, } \\
\text { 1984- } 1985\end{array}$ & 733 men & $\begin{array}{l}760 \text { age- } \\
\text { matched } \\
\text { population } \\
\text { controls }\end{array}$ & $\begin{array}{l}\text { Lifetime } \\
\text { occupational } \\
\text { history from } \\
\text { interview }\end{array}$ & $\begin{array}{l}\text { Ever painter } \\
\text { Duration } \\
\text { (years) } \\
<10 \\
10-19 \\
20-29 \\
\geq 30 \\
>10 \\
<20 \\
>20\end{array}$ & $\begin{array}{l}7 \\
2 \\
5 \\
1 \\
8 \\
9 \\
6\end{array}$ & $\begin{array}{c}1.4(0.5-3.5) \\
\\
1.9(0.36-16.60)^{f} \\
2.8(0.07-62.47)^{f} \\
2.2(0.26-26.67)^{f} \\
0.3(0.01-5.81)^{f} \\
{[1.34} \\
(0.26-6.92)]^{e} \\
{[2.35} \\
(0.44-12.47)]^{e} \\
{[1.18} \\
(0.18-7.64)]^{e}\end{array}$ & $\begin{array}{l}\text { Age, } \\
\text { smoking }\end{array}$ \\
\hline $\begin{array}{l}\text { Ronco et al. } \\
\text { 1988, Italy, } \\
1976-1980\end{array}$ & 126 men & $\begin{array}{l}384 \text { men who } \\
\text { died from } \\
\text { causes other } \\
\text { than from } \\
\text { smoking- } \\
\text { related or } \\
\text { chronic lung } \\
\text { diseases }\end{array}$ & $\begin{array}{l}\text { Lifetime } \\
\text { occupational } \\
\text { history from } \\
\text { interview } \\
\text { with next of } \\
\text { kin }\end{array}$ & Painter & 5 & $1.33(0.43-4.11)$ & $\begin{array}{l}\text { Age, year of } \\
\text { death, } \\
\text { smoking, } \\
\text { other } \\
\text { employment } \\
\text { in suspect } \\
\text { high-risk } \\
\text { occupations }\end{array}$ \\
\hline
\end{tabular}


Table 2. continuation

\begin{tabular}{|c|c|c|c|c|c|c|c|}
\hline $\begin{array}{l}\text { Reference, } \\
\text { location, and } \\
\text { time period }\end{array}$ & $\begin{array}{c}\text { Characteristics } \\
\text { of cases }\end{array}$ & $\begin{array}{c}\text { Characteristics } \\
\text { of controls }\end{array}$ & $\begin{array}{c}\text { Exposure } \\
\text { assessment }\end{array}$ & Exposure & $\begin{array}{l}\text { No. of } \\
\text { exposed } \\
\text { cases }\end{array}$ & OR $(95 \% \mathrm{Cl})$ & $\begin{array}{l}\text { Adjustment } \\
\text { for potential } \\
\text { confounders }\end{array}$ \\
\hline $\begin{array}{l}\text { Vineis et al. } \\
\text { 1988, Analysis } \\
\text { of five case- } \\
\text { control studies } \\
\text { in Louisiana, } \\
\text { Florida, } \\
\text { Pennsylvania, } \\
\text { Virginia, and } \\
\text { New Jersey, } \\
\text { USA, 1970s and } \\
\text { 1980s }\end{array}$ & 2,973 men & 3,210 men & $\begin{array}{l}\text { Lifetime } \\
\text { occupational } \\
\text { history from } \\
\text { interview } \\
\text { with subjects } \\
\text { or next of } \\
\text { kin }\end{array}$ & Painters & 201 & $1.1(0.9-1.4)$ & $\begin{array}{l}\text { Age, birth } \\
\text { cohort, } \\
\text { smoking }\end{array}$ \\
\hline $\begin{array}{l}\text { Lerchen et al. } \\
\text { 1987, N ew } \\
\text { Mexico, USA, } \\
\text { 1980-1982 }\end{array}$ & $\begin{array}{l}771 \text { cases ( } 333 \\
\text { men, } 173 \\
\text { women) }\end{array}$ & $\begin{array}{l}771 \text { controls } \\
\text { ( } 499 \text { men, } 272 \\
\text { women) }\end{array}$ & $\begin{array}{l}\text { Lifetime } \\
\text { occupational } \\
\text { history from } \\
\text { interview }\end{array}$ & $\begin{array}{l}\text { Ever } \\
\text { construction } \\
\text { painters } \\
\text { (men) }\end{array}$ & 9 & $2.7(0.8-8.9)$ & $\begin{array}{l}\text { Age, } \\
\text { ethnicity, } \\
\text { smoking }\end{array}$ \\
\hline $\begin{array}{l}\text { Coggon et al. } \\
\text { 19869, } \\
\text { Cleveland, } \\
\text { Humberside, } \\
\text { Cheshire } \\
\text { counties, United } \\
\text { Kingdom, } \\
\text { 1975-1980 }\end{array}$ & $\begin{array}{l}738 \text { male } \\
\text { bronchial } \\
\text { cancer cases }\end{array}$ & $\begin{array}{l}1,221 \text { other } \\
\text { cancers }\end{array}$ & $\begin{array}{l}\text { Occupation } \\
\text { from mailed } \\
\text { questionnaire }\end{array}$ & $\begin{array}{l}\text { Painters and } \\
\text { decorators }\end{array}$ & 20 & $1.3(0.62-2.72)$ & $\begin{array}{l}\text { Age, } \\
\text { smoking, } \\
\text { residence, } \\
\text { respondent }\end{array}$ \\
\hline $\begin{array}{l}\text { Kjuus et al. } \\
\text { 1986, Norway, } \\
\text { 1979-1983 }\end{array}$ & 176 cases & $\begin{array}{l}176 \text { age- } \\
\text { matched } \\
\text { hospital } \\
\text { controls } \\
\text { excluding } \\
\text { those with } \\
\text { physical or } \\
\text { mental } \\
\text { handicaps, } \\
\text { poor general } \\
\text { health, or } \\
\text { diagnosed with } \\
\text { chronic } \\
\text { obstructive } \\
\text { lung disease }\end{array}$ & $\begin{array}{l}\text { Longest job } \\
\text { held from } \\
\text { interview } \\
\text { and work } \\
\text { site records }\end{array}$ & $\begin{array}{l}\text { Painting, } \\
\text { paper- } \\
\text { hanging } \\
\text { (occupation) }\end{array}$ & 5 & $1.7(0.4-7.3)$ & $\begin{array}{l}\text { Age, } \\
\text { smoking }\end{array}$ \\
\hline $\begin{array}{l}\text { Milne et al. } \\
\text { 1983, Alameda } \\
\text { Country, CA, } \\
\text { USA, } \\
\text { 1958-1962 }\end{array}$ & $\begin{array}{l}925 \text { lung } \\
\text { cancer deaths } \\
\text { ( } 747 \text { men, } \\
178 \text { women) }\end{array}$ & $\begin{array}{l}4,880 \text { deaths } \\
\text { from other } \\
\text { cancers } \\
\text { (except } \\
\text { pancreatic, } \\
\text { bladder, nasal, } \\
\text { kidney, } \\
\text { hematopoietic) } \\
\text { that are not } \\
\text { known to be } \\
\text { strongly } \\
\text { associated with } \\
\text { occupational } \\
\text { risk factors } \\
\text { (reported as } \\
\text { the "reduced } \\
\text { control } \\
\text { group") }\end{array}$ & $\begin{array}{l}\text { Occupation } \\
\text { from death } \\
\text { certificates }\end{array}$ & $\begin{array}{l}\text { Painters } \\
\text { (men) }\end{array}$ & 24 & $1.80(1.09-2.98)^{\mathrm{h}}$ & Age \\
\hline
\end{tabular}


Table 2. continuation

\begin{tabular}{|c|c|c|c|c|c|c|c|}
\hline $\begin{array}{l}\text { Reference, } \\
\text { location, and } \\
\text { time period }\end{array}$ & $\begin{array}{l}\text { Characteristics } \\
\text { of cases }\end{array}$ & $\begin{array}{l}\text { Characteristics } \\
\text { of controls }\end{array}$ & $\begin{array}{l}\text { Exposure } \\
\text { assessment }\end{array}$ & Exposure & $\begin{array}{l}\text { No. of } \\
\text { exposed } \\
\text { cases }\end{array}$ & OR $(95 \% \mathrm{CI})$ & $\begin{array}{l}\text { Adjustment } \\
\text { for potential } \\
\text { confounders }\end{array}$ \\
\hline $\begin{array}{l}\text { Williams et al. } \\
\text { 1977, Atlanta, } \\
\text { GA; } \\
\text { Birmingham, } \\
\text { AL; Colorado; } \\
\text { Dallas-Ft. } \\
\text { Worth, TX; } \\
\text { Detroit, MI; } \\
\text { M inneapolis- } \\
\text { St.Paul, M N; } \\
\text { Pittsburgh, PA; } \\
\text { San Francisco- } \\
\text { Oakland, CA, } \\
\text { USA, Third } \\
\text { National Cancer } \\
\text { Survey }\end{array}$ & 432 cases & $\begin{array}{l}2,173 \text { patients } \\
\text { with cancers } \\
\text { other than } \\
\text { lung, larynx, } \\
\text { oral cavity, } \\
\text { esophagus, } \\
\text { bladder }\end{array}$ & $\begin{array}{l}\text { Main } \\
\text { lifetime } \\
\text { employment } \\
\text { from survey } \\
\text { questionnaire }\end{array}$ & $\begin{array}{l}\text { Painting } \\
\text { (men) }\end{array}$ & 12 & $\begin{array}{c}4.21(1.40-12.65) \\
(p<0.01)\end{array}$ & $\begin{array}{l}\text { Age, race, } \\
\text { education, } \\
\text { tobacco, } \\
\text { alcohol, } \\
\text { geographic } \\
\text { location }\end{array}$ \\
\hline $\begin{array}{l}\text { Viadana et al. } \\
\text { 1976, Decouflé } \\
\text { et al. 1977, } \\
\text { Houten et al. } \\
\text { 1977, Buffalo, } \\
\text { NY, U SA, } \\
\text { 1956-1965 }\end{array}$ & $\begin{array}{l}\text { Lung cancer } \\
\text { cases from } \\
11,591 \text { wite } \\
\text { male cancer } \\
\text { cases }\end{array}$ & $\begin{array}{l}\text { Noncancer } \\
\text { admissions } \\
\text { from the same } \\
\text { cancer } \\
\text { treatment } \\
\text { center }\end{array}$ & $\begin{array}{l}\text { Lifetime } \\
\text { occupation } \\
\text { from } \\
\text { interview } \\
\text { before } \\
\text { diagnosis }\end{array}$ & $\begin{array}{l}\text { Painter } \\
\text { Ever }\end{array}$ & 42 & $1.90(1.32-2.48)$ & $\begin{array}{l}\text { Smoking, } \\
\text { age }\end{array}$ \\
\hline $\begin{array}{l}\text { Breslow et al. } \\
\text { 1954, California, } \\
\text { USA, } \\
\text { 1949- } 1952\end{array}$ & 518 patients & $\begin{array}{l}518 \text { hospital } \\
\text { controls } \\
\text { matched by } \\
\text { hospital, age, } \\
\text { sex, race }\end{array}$ & Interview & $\begin{array}{l}\text { Construction } \\
\text { and } \\
\text { maintenance } \\
\text { painters for } \\
\geq 5 \text { years }\end{array}$ & 22 & $\begin{array}{c}{[1.87} \\
(0.93-3.77)]\end{array}$ & $\begin{array}{l}\text { Hospital, } \\
\text { age, sex, race }\end{array}$ \\
\hline $\begin{array}{l}\text { Wynder and } \\
\text { Graham 1951, } \\
\text { St. Louis, MO, } \\
\text { USA, NG }\end{array}$ & 200 cases & $\begin{array}{l}200 \text { controls } \\
\text { with a chest } \\
\text { disease other } \\
\text { than lung } \\
\text { cancer }\end{array}$ & $\begin{array}{l}\text { Lifetime } \\
\text { occupational } \\
\text { history from } \\
\text { interview }\end{array}$ & $\begin{array}{l}\text { Painter } \geq 5 \\
\text { years within } \\
\text { the last } 40 \\
\text { years }\end{array}$ & 11 & $\begin{array}{c}{[5.76} \\
(1.41-23.44)]\end{array}$ & None \\
\hline
\end{tabular}

NG, not given. Values in brackets were calculated by us.

${ }^{a}$ N onsmokers, subjects who smoked < 400 cigarettes during their lifetime. ${ }^{b}$ BI PS study in Bremen area and Frankfurt/M ain area; GSF study in Nordrhein-Westfalen, Rheinland-Pfalz and Bayern, Saarland, Thuringen, and Sachsen. CFixed-effects model used to calculate a weighted average. ${ }^{\mathrm{d}}$ The study partially overlaps with M orabia et al. 1992 and thus some estimations were used to eliminate the overlap in men and the esti-mated variance was doubled to approximate an adjusted $\mathrm{Cl}$. ${ }^{\mathrm{e}} \mathrm{C}$ alculated using a fixed-effects model. ${ }^{\mathrm{f}}$ Variance was doubled to approximate an adjusted $95 \% \mathrm{Cl}$. 9 Included in the analysis restricted to case-control studies but excluded from the combined meta-analysis because of possible overlap with OPCS 1986 . ${ }^{\mathrm{h}} \mathrm{The} \mathrm{CI}$ was estimated by applying the ratio of reduced/total controls to the observed cell counts reported for the total control group.

An analysis restricted to never-smokers (meta-RR $=2.00 ; 95 \% \mathrm{Cl}, 1.09-3.67 ; \mathrm{I}^{2}=0 \%, \mathrm{p}=$ 0.97) (Kreuzer et al. 2001; Pronk et al. 2009; Zeka et al. 2006) and never-smokers and nonsmokers ( meta-RR $=1.96 ; 95 \% \mathrm{Cl}, 1.15-3.35 ; \mathrm{I}^{2}=0 \%, \mathrm{p}=$ 0.99) (Pohlabeln et al. 2000) demonstrated stronger associations than overall estimates. Regardless of study design, the studies that adjusted for other occupational exposures as well as smoking further strengthened the results (meta-RR $=1.57$; $95 \% \mathrm{Cl}, 1.21-2.04 ; 12=0 \%, p=0.68)$. Because estimates were relatively consistent between individual studies, regardless of study design, it is reasonable to assume that there is no important confounding by tobacco smoking or other occupational exposures among the studies that were not able to adjust for these factors.

Analysis by duration of exposure $(<10$ years vs. $\geq 10$ years, $<20$ years vs. $\geq 20$ years) (Baccarelli et al. 2005; Dalager et al. 1980; Levin et al. 1988; Pronk et al. 2009; Swanson et al. 1993) showed that those exposed $\geq 10$ years (meta-RR $=1.95$; $95 \% \mathrm{Cl}, 1.26-3.02 ;\left.\right|^{2}=0 \%, p=0.63$ ) or $\geq 20$ years ( meta-RR $=2.00 ; 95 \% \mathrm{Cl}, 1.01-3.92 ; \mathrm{I}^{2}=16.4 \%, \mathrm{p}$ $=0.31)$ had a higher risk than those exposed < 10years $\left(\right.$ meta- $\mathrm{RR}=1.13 ; 95 \% \mathrm{Cl}, 0.77-1.65 ; \mathrm{I}^{2}=$ 
Case-control

Wynder and Graham 1951

Breslow et al. 1954

Viadana et al. 1976

Williams et al. 1977

Milne et al. 1983

Kjuus et al. 1986

Lerchen et al. 1987

Vineis et al. 1988

Levin et al. 1988

Ronco et al. 1988

Zahm et al. 1989

Bethwaite et al. 1990

Burns and Swanson 1991

Siemiatycki et al. 1991

M orabia et al. 1992

Notani et al. 1993

Finkelsteins et al. 1995

De Stefani et al. 1996

Muscat et al. 1998

Wunsch-Filho et al. 1998

Jahn et al. 1999; Bruske-Hohlfeld 2000

Pezzotto et al. 1999

Pohlabeln et al. 2000

$M$ atos et al. 2000

Bouchardy et al. 2002

Richiardi et al. 2004

De Stefani et al. 2005

Baccarelli et al. 2005

Zeka et al. 2006

$$
\text { Subtotal }\left(I^{2}=48.4 \%, p=0.002\right)
$$

Cohort and record linkage

OPCS 1958

OPCS 1971

OPCS 1978

OPCS 1986

OPCS 1995

Guralnick et al. 1963

Dunn and Weir 1965

$M$ anck and Henderson 1976

Whorton et al. 1983

Dubrow and Wegman 1984

Guberan et al. 1989

H rubec et al. 1995

Alexander et al. 1996

Van Loon et al. 1997

Boice et al. 1999

Steenland and Palu 1999

Pukkala et al. 2009

Pronk et al. 2009

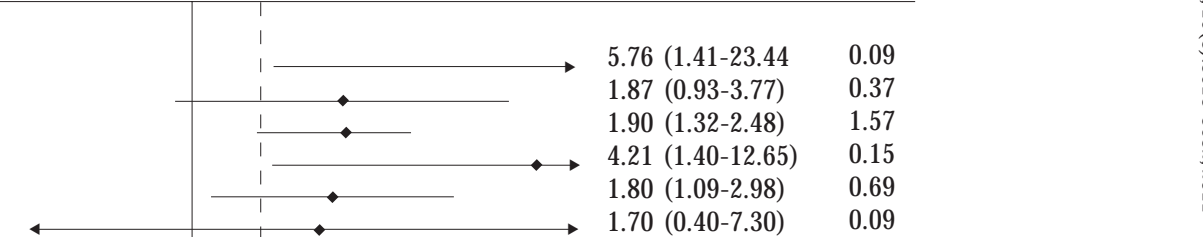

RR $(95 \% \mathrm{Cl})$ weight

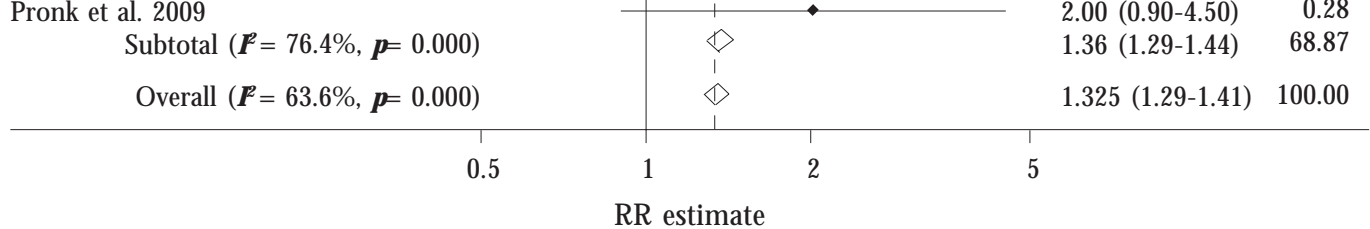

Figure 1. Meta-analysis of all studies assessing lung cancer among persons with occupation as a painter, stratified by study design. Weights are from random-effects analysis. The relative risk estimate for each study is represented by a black diamond, and the horizontal line shows the corresponding $95 \% \mathrm{CI}$. The dashed line marks the combined estimate, and the vertical solid line represents no association. 


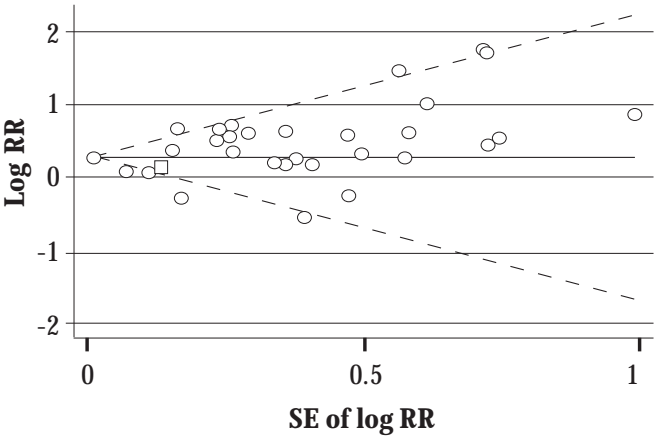

Figure 2. Begg's funnel plot with pseudo- $95 \% \mathrm{Cls}$ to assess publication bias in case-control studies of lung cancer among persons reoporting occupation as a painter.

$0 \%, p=0.46$ ) or $<20$ years ( meta- $R R=1.37 ; 95 \%$ $\mathrm{Cl}, 0.89-2.13 ; 1^{2}=0 \%, p=0.54$ ) (reference category, Oyears of exposure), respectively.

\section{Discussion}

Previous studies demonstrating an increased risk of lung cancer in painters have allowed IARC to classify occupation as a painter as carcinogenic to humans (Group 1) (IARC 1989, in press). This meta-analysis supports the IARC Group 1 classification by demonstrating a 35\% increased risk of lung cancer in painters after adjusting for smoking $\left(\right.$ metaRR $=1.35 ; 95 \% \mathrm{Cl}, 1.21-1.51 ; 1^{2}=41.2 \%$, $p=0.01)$. This association was stronger for population-based case-control studies (meta OR, 1.34; $95 \% \mathrm{Cl}, 1.18-1.51 ;\left.\right|^{2}=25.9 \%, \mathrm{p}=0.16$ ) or studies that adjusted for other potentially confounding occupational exposures (metaRR $=1.57 ; 95 \% \mathrm{Cl}$, $1.21-2.04 ; \mid 2=0 \%, p=0.68)$. Furthermore, exposure-response analyses suggested that the risk increased with duration of employment. Although paint composition or the painting environment could have differed by major geographic region, the results did not vary much when stratified by region (N orth America, Europe, Asia, and South America). This is the first meta-analysisthat demonstrates a relative increase in incidence/mortality from lung cancer in persons occupationally exposed as painters when restricted to never-smokers (and also nonsmokers), as well as demonstrating a statistically significant, positive duration-response relationship.
It is important to note that the interpretation of a meta-SM R (or meta-SIR) for the cohort and record linkage studies is difficult because different referencepopulations were used in each study for the calculation of expected cases or deaths (Rothman et al. 2008). Although the cohort studies of painters could assess possibly higher exposures from longer periods of follow-up, exposure assessment in many of the record linkage studies was often crude: $O$ ccupation as a painter was usually assessed at a single time point in a census and then linked to death registries. Although there can be relatively poor correspondence between occupation recorded on death certificates and in census records (Dubrow and Wegman 1984; Enterline and McKiever 1963; Guralnick 1963; OPCS 1971, 1978) and there is a chance of false-positive results due to multiple testing of occupations in record linkage studies, the SM Rs were remarkably consistent between individual studies, generally ranging between 1.10 and 2.57. This also suggested that the significant results were not likely due to chance. Thus, the approach to combinethe cohort and record linkage study SM Rs for calculating a meta-SM R seemed to bejustified.

In case-control studies, painters may only form a small proportion of the study population, but the full occupational history and additional information on lifestyle factors allowed several studies to adjust for tobacco smoking and some for other occupational carcinogens. An increased lung cancer risk associated with painting was consistently demonstrated in the case-control studies, suggesting that occupation as a painter is a risk factor for lung cancer. Population-based casecontrol studies may be less subject to selection biases than hospital-based case-control studies (Rothman et al. 2008) because there is generally no concern about the appropriate source population if indeed the general population is represented. However, if response rates are low in population controls, this could result in a lack of comparability with cases and therefore be prone to selection biases. A sub-analysis comparing the meta OR of hospital-based and population-based case-control studies showed similar results.

Estimates of the PM R may be biased if the population under study does not share the same distribution of mortality as the standard population used to compute the proportions for categories other than the ones studied (Rothman et al. 2008). However, the proportionate mortality analyses al so showed significantly el evated relative risks for lung cancer in painters within the 
samerange of effect as theanalyses overall and in cohort studies, further suggesting that these re sults remained robust to these biases.

Smoking-adjusted estimates were available for 23 of 29 case-control studies and in only 4 of 18 cohort and record linkagestudies. The robustness of the summary estimates after adjusting for tobacco use, and the higher relative risk in never-smokers, suggest that residual confounding by tobacco use is unlikely and that occupation as a painter is independently associated with the risk of lung cancer.

In women, the meta-RR was similar for all studies (meta-RR $=2.04$; seven studies) ( Jahn et al. 1999; Muscat et al. 1998; OPCS 1958, 1971; Pronk et al. 2009; Pukkala 2009; Zeka et al. 2006) and for studies restricted to never-smokers (meta $R R=2.00$; three studies) (Kreuzer et al. 2001; Pronk et al. 2009; Zeka et al. 2006), further strengthening the evidencethat the resultsarenot confounded by smoking. However, female painters (and never-smoking females) may not actually have a higher risk of lung cancer compared with male painters (meta-RR $=1.37$; 39 studies). The relative risk in women is higher, which may be dueto the fact that women havea lower background lung cancer risk than men (Schottenfeld and Fraumeni 2006).

The robustness of the results is also indicated by the presence of a duration-response relationship, with higher RRs seen for exposure over ed 10 years (meta- $R R=1.95$ ) and ed 20 years ( meta$R R=2.00$ ) compared with those with $<10$ and $<$ 20 years of exposure, respectively (the reference category was no exposure).

Some painters (e.g., in the construction industry) could have been exposed to asbestos. Indeed, a number of studies have shown an increased risk of mesothelioma in painters (Brown et al. 2002; Peto et al. 1995), which is most likely due to occupational asbestos exposure. However, taking into account that the exposure-response relationship for pleural mesothelioma is very different from that for lung cancer, potential asbestos exposure cannot explain all of the increase in lung cancer. Therefore, other suspected carcinogens to which painters are exposed, such as chlorinated solvents, chromium VI compounds, and cadmium compounds (IARC 1987, 1995, 1999, in press; Straif et al. 2009), may also partially explain theincreased risk of lung cancer. Very few studies reported results for specific suspected causative agents. van Loon et al. (1997) reported a positive exposure- response relationship with paint dust and Siemiatycki et al. (1987) found a suggestiveassociation with mineral spirits, whereas Alexander et al. (1996) did not find an increased risk of lung cancer in a cohort of painters and other employees in the aerospace industry exposed to chromium VI compounds.

\section{Conclusion}

There is great variability and complexity in painting environments, which complicates the interpretation of epidemiologic studies of lung cancer risks in painters. Painters are exposed to a wide variety of chemical mixtures, with compositions that change over time. In more recent decades, a number of hazardous chemicals-including benzene, some other solvents, phthalates (plasticizers), and lead oxides- have been reduced or replaced in paint, although thesechemicals are still used in some countries. This trend in reducing exposures to hazardous chemicals in paint has been promoted by the increasing use of waterbased paints and powder coatings. N ew formulations may also contain lower-toxicity solvents, neutralizing agents (e.g., amines), and biocides (IARC 1989, in press). However, this has not yet resulted in lower relative risks for lung cancer in painters, as reported in the more recent observational epidemiologic studies. Theelevated risk of lung cancer may also be partly due to the role that other substances may play in increasing the risk of lung cancer among painters.

Although there was not enough information in the studies provided to assess the association of lung cancer with specific chemical agents encountered in painting, the robustness of the estimates in the subgroup analyses (by sex, region, study design, and controlling for smoking and other occupational exposures) and the stronger associations seen in specific subgroups (by duration of exposure) support the conclusion that occupational exposures in painters are causally associated with the risk of lung cancer. Because several million people are employed as painters worldwide and because lung cancer is the most common cancer in painters, even a modest increase in the relative risk is remarkable. It is important for cancer control and prevention to design studies with better exposure assessment to identify the underlying carcinogenic agents encountered in painting. 


\section{Acknowledgements}

We thank D. Russell, K. Abdedayem, S. Egraz, and S. Grant for technical assistance. The authors declarethey haveno competing financial interests.

\section{References}

Alexander BH, Checkoway H, Wechsler L, Heyer NJ, Muhm JM, O'Keeffe TP. 1996. Lung cancer in chromate-exposed aerospace workers. J Occup Environ Med 38:1253-1258.

Baccarelli A, Tretiakova M, Gorbanev S, Lomtev A, Klimkina I, Tchibissov V, et al. 2005. Occupation and lung cancer risk in Leningrad Province, Russia. M ed Lav 96:142-154.

Bethune A, Harding S, Scott A, Filakati H. 1995. M ortality of longitudinal study 1971 and 1981 census cohorts. In: Occupational Health Decennial Suplement. Series DS10. London:H M SO, 103-126.

Bethwaite PB, Pearce N, Fraser J. 1990. Cancer risks in painters: study based on the $\mathrm{N}$ ew Zealand Cancer Registry. $\mathrm{Br} J$ Ind Med 47:742-746.

Boffetta P, Trichopoulos D. 2002. Cancer of the lung, larynx and pleura. In: Textbook of Cancer Epidemiology. New York:Oxford University Press, 248280.

Boice JD Jr, Marano DE, Fryzek JP, Sadler CJ, M Laughlin JK. 1999. M ortality among aircraft manufacturing workers. Occup Environ M ed 56:581-597.

Bouchardy C, Schuler G, Minder C, Hotz P, Bousquet A, Levi $F$, et al. 2002. Cancer risk by occupation and socioeconomic group among men-a study by the Association of Swiss Cancer Registries. Scand J Work Environ Health 28:1-88.

Bradburn MJ. 2004. Updated and New Commands for Meta-analysis in Stata. Available: http:// w w w. medepi.net/meta/ software/ Bradburn_metan_updates.pdf [accessed 24 August 2009].

Breslow L, Hoaglin L, Rasmussen G, Abrams HK. 1954. Occupations and cigarette smoking as factors in lung cancer. Am J Public Health 44(2):171-181.

Brown LM, Moradi T, Gridley G, Plato N, Dosemeci $M$, Fraumeni JF Jr. 2002. Exposures in the painting trades and paint manufacturing industry and risk of cancer among men and women in Sweden. J Occup Environ Med 44:258-264.

Brüske-Hohlfeld I, M ohner M, Pohlabeln H, Ahrens W, Bolm-Audorff U, Kreienbrock L, et al. 2000. Occupational lung cancer risk for men in Germany: results from a pooled case-control study. Am J Epidemiol 151:384-395.

Burns PB, Swanson GM. 1991. The Occupational Cancer Incidence Surveillance Study (OCISS): risk of lung cancer by usual occupation and industry in the Detroit metropolitan area. Am J Ind M ed 19:655671.

Carstensen JM, Pershagen G, Eklund G. 1988. Smoking-adjusted incidence of lung cancer among Swedish men in different occupations. Int J Epidemiol 17:753-758.
Coggon D, Pannett B, Osmond C, Acheson ED. 1986. A survey of cancer and occupation in young and middle aged men. I. Cancers of the respiratory tract. $\mathrm{Br}$ $J$ Ind M ed 43(5):332-338.

Dalager NA, M ason TJ, Fraumeni JF Jr, Hoover R, Payne WW. 1980. Cancer mortality among workers exposed to zinc chromate paints. J Occup Med 22(1):25-29.

De Stefani E, Boffetta P, Brennan P, Deneo-Pellegrini $H$, Ronco A, Gutierrez LP. 2005. Occupational exposures and risk of adenocarcinoma of the lung in Uruguay. Cancer Causes Control 16:851-856.

De Stefani E, Kogevinas M, Boffetta P, Ronco A, M endilaharsu M. 1996. Occupation and the risk of lung cancer in Uruguay. Scand J Work Environ Health 22:346-352.

Decouflé P, Stanislawczyk K, Houten L, Bross IDJ, Viadana $E$, eds. 1977. A Retrospective Survey of Cancer in Relation to Occupation. DHEW (NIOSH) Publication No. 77-178. Cincinnati, $\mathrm{OH}: \mathrm{N}$ ational Institute for Occupational Safety and Health.

Deeks JJ, Altman DG, Bradburn MJ. 2005. Statistical methods for examining heterogeneity and combining results from several studies in meta-analysis. In: Systematic Reviews in H ealth: Care M eta-Analysis Context. London:BMJ Books, 285-312.

DerSimonian R, Laird N. 1986. M eta-analysis in clinical trials. Control Clin Trials 7:177-188.

Dubrow R, Wegman DH. 1984. Cancer and occupation in M assachusetts: a death certificate study. Am J Ind Med 6:207-230.

Dunn JE Jr, Weir JM. 1965. Cancer experience of several occupational groups followed prospectively. Am J Public Health Nations Health 55:1367-1375.

Enterline PE, McKiever M F. 1963. Differential mortality from lung cancer by occupation. J Occup Med 5:283-290.

Finkelstein M M. 1995. Occupational associations with lung cancer in two Ontario cities. Am J Ind Med 27:127-136.

Gubéran E, U sel M, Raymond L, Tissot R, Sweetnam PM. 1989. Disability, mortality, and incidence of cancer among Geneva painters and electricians: a historical prospective study. Br J Ind Med 46:1623.

Guralnick L, ed. 1963. M ortality by Occupation Level and Cause of Death Among Men 20 to 64 Years of Age: USA, 1950. Washington, DC:U.S. Department of Health, Education, and Welfare.

Higgins JP, Thompson SG. 2002. Quantifying heterogeneity in a meta-analysis. Stat Med 21:1539-1558.

Houten L, Bross IDJ, Viadana E, Sonnesso G. 1977. Occupational cancer in men exposed to met als. Adv Exp Med Biol 91:93-102. 
H rubec A, Blair A, Vaught J, eds. 1995. Mortality Risks by Occupation among US Veterans of Known Smoking Status 1954-1980. Washington, DC:National Cancer Institute.

H uedo-M edina TB, Sanchez-M eca J, M arin-M artinez F, Botella J. 2006. Assessing heterogeneity in metaanalysis: Q statistic or 12 index? Psychol Methods 11:193-206.

IARC (International Agency for Research on Cancer). 1987. Overall Evaluations of Carcinogenicity: An Updating of IARC Monographs Volumes 1 to 42.IARC M onogr Eval Carcinog Risk Hum Suppl 7:1-440.

IARC (International Agency for Research on Cancer). 1989. Occupational exposures in paint manufacture and painting. IARC M onogr Eval Carcinog Risk Hum 47:329-442.

IARC (International Agency for Research on Cancer). 1995. Trichloroethylene. IARC Monogr Eval Carcinog Risk Hum 63:75-158.

IARC (International Agency for Research on Cancer). 1999. Dichloromethane. IARC Monogr Eval Carcinog Risk Hum 71:251-315.

IARC (International Agency for Research on Cancer). 2001. Globocan 2000: Cancer Incidence and Mortality Worldwide, IARC Cancer Bases No. 5 (Ferlay J, Bray FI, Parkin DM, Pisani P, eds). Lyon, France:IARC Press.

IARC (International Agency for Research on Cancer). 2003. World Cancer Report (Stewart B, Kleihues P, eds). Lyon, France:IARC Press. Available: http:// www.iarc.fr/en/publications/pdfs-online/wcr/2003/ index.php [accessed 31 August 2009].

IARC (International Agency for Research on Cancer). 2008. World Cancer Report (Boyle P, Levin B, eds). Lyon, France:IARC Press, 9-510. Available: http:// www.iarc.fr/en/publications/pdfs- online/wcr/2008/ index.php [accessed 31 August 2009].

IARC (International Agency for Research on Cancer). In press. Shift-work, painting and fire-fighting. IARC Monogr Eval Carcinog Risks Hum 98.

Jahn I, Ahrens W, Bruske-H ohlfeld I, Kreuzer M, M ohner M, Pohlabeln H, et al. 1999. Occupational risk factors for lung cancer in women: results of a casecontrol study in Germany. Am J Ind Med 36:90100.

Kjuus H, Skjaerven R, Langard S, Lien JT, Aamodt T. 1986. A case-referent study of lung cancer, occupational exposures and smoking. I. Comparison of title-based and exposure-based occupational information. Scand J Work Environ Health 12(3):193202.

Kreuzer M, Gerken M, Kreienbrock L, Wellmann J, Wichmann HE. 2001. Lung cancer in lifetime nonsmoking men-results of a case-control study in Germany. Br J Cancer 84:134-140.

Lerchen ML, Wiggins CL, Samet JM . 1987. Lung cancer and occupation in New M exico. J Natl Cancer Inst 79:639-645

Levin LI, Zheng W, Blot WJ, Gao YT, Fraumeni JF Jr. 1988. Occupation and lung cancer in Shanghai: a case-control study. Br J Ind Med 45(7):450-458.

Lewis S, Clarke M. 2001. Forest plots: trying to see the wood and the trees. BMJ 322:1479-1480.

Logan WP. 1982. Cancer Mortality by Occupation and Social Class 1851-1971. IARC Sci Publ 36:1-253.
Matos EL, Vilensky M, M irabelli D, Boffetta P. 2000. Occupational exposures and lung cancer in Buenos Aires, Argentina. J Occup Environ Med 42:653659.

Menck HR, Henderson BE. 1976. Occupational differences in rates of lung cancer. J Occup Med 18:797801.

Milne KL, Sandler DP, Everson RB, Brown SM. 1983. Lung cancer and occupation in Alameda County: a death certificate case-control study. Am J Ind Med 4:565- 575 .

Morabia A, Markowitz S, Garibaldi K, Wynder EL. 1992. Lung cancer and occupation: results of a multicentre case-control study. $\mathrm{Br}$ J Ind Med 49:721-727.

Muscat JE, Stellman SD, Richie JP Jr, Wynder EL. 1998. Lung cancer risk and workplace exposures in black men and women. Environ Res 76:78-84.

$\mathrm{N}$ ational Center for Biotechnology Information. 2009. PubM ed. Available: http://www.ncbi.nlm.nih.gov/ pubmed/ [accessed 31 August 2009].

Notani PN, Shah P, Jayant K, Balakrishnan V. 1993. Occupation and cancers of the lung and bladder: a case-control study in Bombay. Int J Epidemiol 22:185-191.

OPCS (Office of Population Censuses and Surveys), ed. 1958. The Registrar General's Decennial Supplement, England and Wales 1951: Occupational Mortality Tables. Pt II, Vol 2. London:H M SO.

OPCS (Office of Population Censuses and Surveys), ed. 1971. The Registrar General's Decennial Supplement, England and Wales 1961: Occupational Mortality Tables. London:H M SO.

OPCS (Office of Population Censuses and Surveys), ed. 1978. Occupational Mortality. The Registrar General's Decennial Supplement, England and Wales 1970-1972: DS N o. 1. London:H M SO.

OPCS (Office of Population Censuses and Surveys), ed. 1986. O ccupational Mortality 1979-80, 1982-83, Great Britain, Decennial Supplement. DS No. 6. London:H M SO.

OPCS (Office of Population Censuses and Surveys). 1995. The Registrar General's Health and Safety Executive. Occupational Health: Decennial Supplement (Drever F, ed). DS N o. 10. London:H M SO.

Petersen GR, Milham SJ, eds. 1980. Occupational Mortality in the State of California 1959-61. Publication No. 80-104. Cincinnati, $\mathrm{OH}: \mathrm{N}$ ational Institute for Occupational Safety and $\mathrm{H}$ ealth.

Peto J, Hodgson JT, Matthews FE, Jones JR. 1995. Continuing increase in mesothelioma mortality in Britain. Lancet 345:535-539.

Peto R, Lopez AD, Boreham J, Heath C, Thun M, eds. 1994. Mortality from Tobacco in Developed Countries, 1950-2000. Oxford, UK:Oxford University Press.

Pezzotto SM, Poletto L. 1999. Occupation and histopathology of lung cancer: a case-control study in Rosario, Argentina. Am J Ind M ed 36:437-443.

Pohlabeln H, Boffetta P, Ahrens W, Merletti F, Agudo A, Benhamou E, et al. 2000. Occupational risks for lung cancer among nonsmokers. Epidemiology 11:532-538.

Pronk A, Coble J, Ji BT, Shu XO, Rothman N, Yang G, et al. 2009. Occupational risk of lung cancer among lifetime non-smoking women in Shanghai, China. Occup Environ Med 66:672-678. 
Pukkala E. 2009. Occupation and cancer. Follow-up of 15 million people in five $\mathrm{N}$ ordic Countries. Acta Oncol 48:646-790.

Richiardi L, Boffetta P, Simonato L, Forastiere F, Zambon P, Fortes C, et al. 2004. Occupational risk factors for lung cancer in men and women: a population-based case-control study in Italy. Cancer Causes Control 15:285-294.

Ronco G, Ciccone G, Mirabelli D, Troia B, Vineis P. 1988. Occupation and lung cancer in two industrialized areas of northern Italy. Int J Cancer 41(3):354358.

Rothman KJ, Greenland S, Lash TL, eds. 2008. Modern Epidemiology. Philadelphia:Lippincott, Williams and Wilkins.

Schottenfeld D, Fraumeni J, eds. 2006. Cancer Epidemiology and Prevention. New York:Oxford University Press. Siemiatycki J, ed. 1991. Risk Factors for Cancer in the Workplace. Boca Raton, FL:CRC Press.

Siemiatycki J, Dewar R, Nadon L, Gerin M, Richardson L, Wacholder S. 1987. Associations between several sites of cancer and twelve petroleum-derived liquids. Results from a case-referent study in Montreal. Scand J Work Environ Health 13:493-504.

Siemiatycki J, Richardson L, Straif K, Latreille B, Lakhani R, Campbell S, et al. 2004. Listing occupational carcinogens. Environ $\mathrm{H}$ ealth Perspect 112:1447-1459.

Steenland K, Palu S. 1999. Cohort mortality study of 57,000 painters and other union members: a 15 year update. Occup Environ Med 56(5):315-321.

Stockwell HG, Matanoski GM. 1985. A case-control study of lung cancer in painters. J Occup Med 27(2):125-126.

Straif K, Baan R, Grosse Y, Secretan B, El GF, Bouvard $V$, et al. 2007. Carcinogenicity of shift-work, painting, and fire-fighting. Lancet Oncol 8:1065-1066.

Straif K, Benbrahim-Tallaa L, Baan R, Grosse Y, Secretan B, El Ghissassi F, et al. 2009. A review of human carcinogens- part C: met als, arsenic, dusts, and fibres. Lancet Oncol 10:453-454.

Sutton AJ, Abrams KR, Jones DR, Sheldon TA, Song F, eds. 2000. M ethods for M eta-Analysis in Medical Research. New York:Wiley.

Swanson GM, Lin CS, Burns PB. 1993. Diversity in the associa-tion between occupation and lung cancer among black and white men. Cancer Epidemiol Biomarkers Prev 2:313-320.

Taeger D, Sun Y, Keil U, Straif K. 2000. A stand-alone Windows applications for computing exact personyears, standardized mortality ratios and confidence intervals in epidemiological studies. Epidemiology 11:607-608. van Loon AJ, Kant IJ, Swaen GM, Goldbohm RA, KremerAM, van den Brandt PA. 1997. Occupational exposure to carcinogens and risk of lung cancer: results from The Netherlands cohort study. Occup Environ Med 54:817-824.

Viadana E, Bross IDJ, Houten L. 1976. Cancer experience of men exposed to inhalation of chemicals or to combustion products. J Occup M ed 18:787-792.

Vineis P, Thomas T, Hayes RB, Blot WJ, Mason TJ, Pickle LW, et al. 1988. Proportion of lung cancers in males, due to occu-pation, in different areas of the USA. Int J Cancer 42:851-856.

Whorton MD, Schulman J, Larson SR, Stubbs HA, AustinD. 1983. Feasibility of identifying high-risk occupations through tumor registries. J Occup M ed 25:657-660.

Williams RR, Stegens NL, Goldsmith JR. 1977. Associations of cancer site and type with occupation and industry from the Third National Cancer Survey Interview. J Natl Cancer Inst 59:1147-1185.

Wünsch-Filho V, M oncau JE, M irabelli D, Boffetta P. 1998. Occupational risk factors of lung cancer in Sao Paulo, Brazil. Scand J Work Environ Health 24:118-124.

Wynder EL, Graham EA. 1951. Etiologic factors in bronchiogenic carcinoma with special reference to industrial exposures. Report of eight hundred fiftyseven proved cases. Arch Ind Hyg Occup M ed 4:221235.

Zahm SH, Brownson RC, Chang JC, Davis JR. 1989. Study of lung cancer histologic types, occupation, and smoking in Missouri. Am J Ind Med 15:565578.

Zeka A, M annetje A, Zaridze D, Szeszenia-Dabrowska $\mathrm{N}$, RudnaiP, Lissowska J, et al. 2006. Lung cancer and occu-pation in nonsmokers: a multicenter casecontrol study in Europe. Epidemiology 17:615-623.

Received 31 August 2009 Accepted 220 ctober 2009 